\title{
House allocation with overlapping generations
}

\author{
Citation for published version (APA):
}

Kurino, M. (2011). House allocation with overlapping generations. METEOR, Maastricht University School of Business and Economics. METEOR Research Memorandum No. 032 https://doi.org/10.26481/umamet.2011032

Document status and date:

Published: 01/01/2011

DOI:

10.26481/umamet.2011032

Document Version:

Publisher's PDF, also known as Version of record

\section{Please check the document version of this publication:}

- A submitted manuscript is the version of the article upon submission and before peer-review. There can be important differences between the submitted version and the official published version of record.

People interested in the research are advised to contact the author for the final version of the publication, or visit the DOI to the publisher's website.

- The final author version and the galley proof are versions of the publication after peer review.

- The final published version features the final layout of the paper including the volume, issue and page numbers.

Link to publication

\footnotetext{
General rights rights.

- You may freely distribute the URL identifying the publication in the public portal. please follow below link for the End User Agreement:

www.umlib.nl/taverne-license

Take down policy

If you believe that this document breaches copyright please contact us at:

repository@maastrichtuniversity.nl

providing details and we will investigate your claim.
}

Copyright and moral rights for the publications made accessible in the public portal are retained by the authors and/or other copyright owners and it is a condition of accessing publications that users recognise and abide by the legal requirements associated with these

- Users may download and print one copy of any publication from the public portal for the purpose of private study or research.

- You may not further distribute the material or use it for any profit-making activity or commercial gain

If the publication is distributed under the terms of Article $25 \mathrm{fa}$ of the Dutch Copyright Act, indicated by the "Taverne" license above, 


\section{Maastricht University}

Morimitsu Kurino

House allocation with overlapping generations

$\mathrm{RM} / 11 / 032$

\section{METEOR}

Maastricht University School of Business and Economics

Maastricht Research School of Economics

of Technology and Organization

P.O. Box 616

NL - 6200 MD Maastricht

The Netherlands 


\title{
House Allocation with Overlapping Generations*
}

\author{
Morimitsu Kurino $^{\dagger}$
}

First Draft: October 2008

This Draft: May 2011

\begin{abstract}
Many real-life applications of house allocation problems are dynamic. For example, each year college freshmen move in and seniors move out of on-campus housing. Each student stays on campus for only a few years. A student is a "newcomer" in the beginning and then becomes an "existing tenant." Motivated by this observation, we introduce a model of house allocation with overlapping generations. In terms of a dynamic rule without monetary transfers, we examine two static rules of serial dictatorship (SD) and top trading cycles (TTC), both of which are based on an ordering of agents and give a higher-order agent a more advantageous position in the assignment procedure. We support a seniority-based SD rule by showing its dynamic Pareto efficiency. Similarly, we support a seniority-based TTC rule under timeinvariant preferences by showing its dynamic Pareto efficiency and incentive compatibility.

Keywords: house allocation, overlapping generations, seniority-based rules, serial dictatorship, top trading cycles

JEL classification: C71; C78; D71; D78
\end{abstract}

*This paper is based on Chapter 4 of my Ph.D. dissertation submitted to the University of Pittsburgh. I am very grateful to Onur Kesten and Ted Temzelides, especially to M. Utku Ünver, for their guidance and support. I am also grateful to Masaki Aoyagi, Sourav Bhattacharya, Andreas Blume, Oliver Board, Lars Ehlers, Tim Hister, Eiichi Miyagawa, David Parkes, William Thomson, and in particular to an advisory editor of the journal and two anonymous referees whose comments and suggestions improved the paper. I also thank seminar participants at Bonn, Max Plank Institute of Economics, Sophia U., 2010 Japanese Economic Association annual meeting, and the 2010 Econometric Society World Congress for their comments. All errors are my own responsibility.

${ }^{\dagger}$ Maastricht University, Department of Economics, P.O. Box 616, 6200 MD Maastricht, The Netherlands; m.kurino@maastrichtuniversity.nl 


\section{Introduction}

The static allocation problem of assigning indivisible goods, called "houses," to agents without monetary transfers has been studied extensively and applied to real-life markets such as on-campus housing for college students (Abdulkadiroğlu and Sönmez, 1999; Chen and Sönmez, 2002; Guillen and Kesten, 2010), kidney exchanges for patients (Roth, Sönmez, and Ünver, 2004, 2005), and school choice for U.S. public schools (Abdulkadiroğlu and Sönmez, 2003). Until now, there have been few attempts to analyze dynamic house allocation problems. ${ }^{1}$

Considering the dynamic aspects of the issue enables us to study features of the allocation problem that cannot be captured by static models. For example, in the case of on-campus housing for college students, each year freshmen move in and graduating seniors leave. Each student stays on campus for only a few years. A student is a "newcomer" in the beginning and then becomes an "existing tenant." 2 In general, students are overlapping. In this structure, it is not always dynamically Pareto efficient to have a static Pareto-efficient matching in each period.

To illustrate this point, suppose that in the first period $t=1$, there is one agent $a^{0}$, called an initial existing tenant, who has come before the market starts and lives there only in this period. Moreover, in each period $t \geq 1$, one agent $a^{t}$ arrives to live in a house in periods $t$ and $t+1$. In each period $t$, there is an existing tenant $a^{t-1}$, who has come in the previous period, and a newcomer $a^{t}$. There are two durable houses $h_{1}$ and $h_{2}$. Each agent prefers $h_{1}$ to $h_{2}$, and $\left(h_{2}, h_{1}\right)$ to $\left(h_{1}, h_{2}\right){ }^{3}$ Consider the following plan:

\begin{tabular}{|c|c|c|c|c|c|}
\hline & $t=1$ & $t=2$ & $t=3$ & $t=4$ & $\cdots$ \\
\hline$a^{0}$ & $h_{2}$ & & & & \\
\hline$a^{1}$ & $h_{1}$ & $h_{2}$ & & & \\
\hline$a^{2}$ & & $h_{1}$ & $h_{2}$ & & \\
\hline$a^{3}$ & & & $h_{1}$ & $h_{2}$ & \\
\hline$\vdots$ & & & & $\vdots$ & $\vdots$ \\
\hline
\end{tabular}

In each period, an existing tenant is assigned $h_{2}$ and a newcomer is assigned $h_{1}$. This plan is Pareto efficient for each period's static market. However, consider an infinite exchange between an

\footnotetext{
${ }^{1}$ See recent exceptions: Abdulkadiroğlu and Loertscher (2007), Bloch and Cantala (2008), Kamijo and Kawasaki (2010), and Ünver (2010).

${ }^{2}$ In the literature of house allocation with existing tenants (Abdulkadiroğlu and Sönmez, 1999), existing tenants have the property rights on houses and newcomers do not. Contrary to this usage, in this paper, given any period of time, existing tenants are the agents who have come to the market in a previous period, while newcomers are those who have just arrived. Property rights can be endogenous in a dynamic rule. We introduce and study a dynamic rule with specific property rights transfer, i.e., a spot rule with property rights transfer, in which in any peirod of time, existing tenants have the property rights on a previously lived-in house and newcomers do not.

${ }^{3}$ For example, $\left(h_{2}, h_{1}\right)$ is a consumption path where an agent consumes house $h_{2}$ in the first period, and $h_{1}$ in the next. Note that this preference violates the discounted utility model. However, considering a critique of the discounted utility model as reviewed by Frederick, Lowenstein, and O'Donoghue (2002), we allow for any preference relation on $\left\{h_{1}, h_{2}\right\} \times\left\{h_{1}, h_{2}\right\}$ in this paper. See footnote 16 for further discussion.
} 
existing tenant and a newcomer in each period, where an existing tenant exchanges her house $h_{2}$ for the newcomer's house $h_{1}$. As a result, the initial existing tenant is assigned $h_{1}$, and each of the other agents is assigned $\left(h_{2}, h_{1}\right)$. Every agent prefers this new plan to the original. Thus, the original plan is not dynamically Pareto efficient.

Many universities in the United States use a variant of the so-called random serial dictatorship rule to allocate dormitory rooms. ${ }^{4}$ This rule randomly orders the agents and then applies the serial dictatorship (SD) rule: the first agent is assigned her top choice, and the next agent is assigned her top choice among the remaining rooms, and so on. This ordering is not entirely random, but rather depends on seniority. That is, existing tenants are favored over newcomers.

In the previous example, consider period orderings that order a newcomer $a^{t}$ as the first and an existing tenant $a^{t-1}$ as the second in each period. Applying an SD rule in each period, we obtain the same plan as indicated in the previous table. As we saw, this plan is not dynamically Pareto efficient. By contrast, consider other period orderings that order an existing tenant first, and a newcomer next. The plan by the SD rule with these orderings Pareto dominates the original and is dynamically Pareto efficient. That is, when we apply the SD rule each period, the orderings based on seniority perform well in terms of dynamic Pareto efficiency.

The subject of this paper is to present a new dynamic framework for the house allocation problem by considering an overlapping generations structure, ${ }^{5}$ and to analyze the impact of orderings on dynamic Pareto effciency and the incentive compatibility of rules. To our knowledge, we are the first to offer the rationale for seniority-based rules in terms of the positive properties. ${ }^{6}$

Our model extends the standard overlapping generations (OLG) model $^{7}$ to the house allocation problem. Time is discrete and lasts forever. There are finitely many durable houses ${ }^{8}$ that are collectively owned, but only initial existing tenants may have the right to live in a house in the first period. In each period, finitely many agents arrive to stay for two periods, while the oldest agents leave the market. Each agent consumes one house in each period. Each agent has a time-separable preference over consumption paths, consisting of period preferences. Her preference does not evolve over time: during her stay, she has the the same preference as she had upon arrival. However, we do allow period preferences to vary across periods. A real-life example that fits this model is that of dormitory room-assignment for on-campus housing and room assignment for apartment management companies.

\footnotetext{
${ }^{4}$ We will mention some of real-life examples later in this section.

${ }^{5}$ Block and Cantala (2008) independently consider a similar model to ours. See the Section on Related literature for the difference.

${ }^{6}$ There are almost no papers on the importance of seniority-based rules, but an exception is Sönmez and Ünver (2005). They show in a house allocation problem with existing tenants that the core rule from random endowments of vacant houses to newcomers, which is a natural appealing approach based on Abdulkadiroğlu and Sönmez (1998), is extremely biased in the sense that it is equivalent to a stochastic AS-TTC rule favoring newcomers.

${ }^{7}$ See Samuelson (1958), or Ljungqvist and Sargent (2004).

${ }^{8}$ In the standard OLG model, a consumption good is not durable but perishable. Another equivalent assumption of durability of houses in our OLG model is that houses are perishable but the same supply of houses "falls from heaven" every period, as pointed out by a referee. But we do not take this interpretation, because our main applications are dormitory rooms and apartments as indivisible objects that are considered to be durable.
} 
We focus on a particular dynamic rule, called a spot rule: each agent reveals her period preferences instead of the full preference. Based on the reported period-preference profile, a spot rule chooses a plan. Depending on whether property rights are transferable, we analyze the two types of spot rules: In a spot rule with property rights transfer, the houses occupied by the oldest agent become vacant in the next period, but those occupied by the other agents become their properties that they can trade or continue to occupy. On the other hand, a spot rule without property rights transfer has no transfer of property rights.

At any point in time, our spot rule without property rights transfer resembles a house allocation problem (Hylland and Zeckhauser, 1979) where no agent has property rights on any house. The representative rule in theory and practice is a (static) random serial dictatorship (RSD) (Abdulkadiroğlu and Sönmez, 1998). Some colleges, such as Davidson College, Lafayette College, and St. Olaf College, use a seniority-based RSD spot rule with the requirement that all students participate every year. A serial dictatorship (SD) spot rule is a spot rule without property rights transfer in which an SD static rule is applied in each period, given period orderings. As we saw in the previous example, period orderings that favor newcomers do not always induce a dynamically Pareto-efficient plan (Theorem 2). On the other hand, a specific period ordering that favors existing tenants does induce a dynamically Pareto-efficient plan (Theorem 3).

We also introduce a notion of "acceptability": an acceptable spot rule always induces a plan under which each existing tenant finds her current house at least as desirable as her previous one. ${ }^{9}$ The importance of acceptability comes from observations of on-campus housing and apartment assignments.

In on-campus housing, the RSD rule is rarely used, although it is simple, Pareto efficient, and strategy-proof (Svensson, 1994). Rather, many universities use its modified rule, called an $R S D$ rule with squatting rights, where existing tenants either keep their current rooms or give up them and participate in the RSD rule. This seniority-based rule is used at Northwestern University, the University of Michigan, and the University of Pittsburgh, among others. Students in these universities can choose to stay off campus. Even colleges that require all students to live on campus use this seniority-based rule, for example, Gordon College, Guilford College, and Lawrence University. This rule is acceptable for existing tenants who keep their current rooms, but not acceptable for those entering the RSD rule who might end up with a worse house; i.e., it is not (ex-post) individually rational. The main reason for its $\mathrm{use}^{10}$ is that universities want to keep

\footnotetext{
${ }^{9}$ The acceptability reminds us of the Ratchet effect-the tendency of performance standards in an incentive system to be adjusted upward after a particularly good performance (for example, see Milgrom and Roberts (1992)). I thank William Thomson for pointing this out.

${ }^{10}$ James Earle, Assistant Vice Chancellor for Business at the University of Pittsburgh, gave me the following official reason: "The goal of the Department of Housing is, first and foremost, customer satisfaction. By allowing students the opportunity to retain a room they like, we are guaranteeing the satisfaction of these returning customers. Furthermore, if these students were forced out of their room, they could not only become a dissatisfied customer, if they then get a room they don't like, but they could also decide to live off campus and become someone else's customer. Why risk the loss of revenue, when you have the potential to have a satisfied customer simply by allowing them to retain their room?"
} 
students on campus, which makes the universities financially less risky. Thus, an acceptable rule is desirable to both students and universities.

Turning to apartment assignment, companies face a legal contract where a tenant is protected from eviction during the lease so long as the tenant does not violate any substantial provision or any local housing laws or code. ${ }^{11}$ Thus, apartment assignment must also be acceptable.

The above two observations also motivate us to examine a spot rule with property rights transfer. At any point in time, this spot rule resembles a static house allocation problem with existing tenants (Abdulkadiroğlu and Sönmez, 1999) in which there are newcomers (agents without property rights) and existing tenants (agents with property rights). In a static context, Abdulkadiroğlu and Sönmez (1999) show that the RSD rule with squatting rights is neither (ex-post) individually rational nor statically Pareto efficient. For this reason, they propose a rule based on the Gale's top trading cycles (TTC) rule (Shapley and Scarf, 1974), referred to as $A S-T T C$ rule. ${ }^{12}$ This rule restores static Pareto efficiency that the RSD rule with squatting rights lacks, while satisfying individual rationality and strategy-proofness.

Any SD spot rule is not acceptable, since all houses that an existing tenant finds at least as desirable as her previously owned one can be obtained by higher-order agents. On the other hand, acceptability is always achieved when in each period an existing tenant is assigned the property rights of her previously owned house and is then assigned a house induced by an individually rational static rule. Thus, we consider the spot rule with property rights transfer where we apply an individually rational AS-TTC rule in each period. This rule is called a TTC spot rule.

However, we get the impossibility result (Theorem 1) in which no spot rule is dynamically Pareto efficient and acceptable. Therefore, any TTC spot rule is not dynamically Pareto efficient although it induces a statically Pareto-efficient matching in each period. By restricting the problem to the time-invariant preference domain, where the preference of each agent consists of identical period preferences, we avoid the impossibility result and show that a specific seniority-based TTC spot rule is both dynamically Pareto efficient and acceptable, as well as incentive compatible (Theorems 5 and 7). But a non-seniority based rule, i.e., a TTC spot rule favoring newcomers, is neither dynamically Pareto efficient nor incentive compatible (Theorems 6 and 8).

We emphasize that in the time-invariant preference domain, a TTC spot rule is not just a repetition of an AS-TTC static rule but has distinct features: ${ }^{13}$ We have entry and exit of agents with different preferences in each period. Also, property rights are endogenous.

\footnotetext{
${ }^{11}$ For example, see http://www.housingnyc.com/index.html for the rent guidelines by New York City Rent Guidelines Board.

${ }^{12}$ AS-TTC rule works as follows: Given the ordering of agents, assign agents their favorite houses one-at-a-time following their ordering and whenever an agent demands the house of an existing tenant, modify the ordering by inserting the existing tenant at the top. Whenever a loop of existing tenants forms, assign each of them the house she demands and proceed.

${ }^{13}$ Although the consistency concept in a static model takes care of the variable population, it does not allow endogenous property rights. For the consistency concept, see Sönmez and Ünver (2010) for a house allocation problem with existing tenants, Ergin (2000) for a house allocation problem, and Thomson (2008) for the various resource allocation problems.
} 
Our main theoretical contributions are to show, when one applies an SD or AS-TTC rule that is statically Pareto-efficient and strategy-proof in each period, how and whether such static properties carry over to dynamic Pareto efficiency and incentive compatibility in a dynamic matching model. We show that this question depends on whether the static rules are seniority based or not. To this end, after describing the model in Section 2, we examine a general spot rule in Section 3. Then, we contrast seniority-based rules with the non-seniority-based ones of SD rules and TTC rules in Sections 4 and 5, respectively. In Section 6, we discuss the case where agents live for at least three periods. Finally, in the last section we discuss our results.

\subsection{Related literature}

There is an extensive literature on static house allocation problems. Pápai (2000a) introduces hierarchical exchange rules, which are similar to but wider than the AS-TTC rules by Abdulkadiroğlu and Sönmez (1999) in that Pápai's rules accommodate a more general than theirs and uses the idea of Gale's TTC algorithm. Her rules characterize a class of Pareto-efficient, reallocation-proof, and group strategy-proof rules. Recently, Pycia and Ünver (2010) proposed a wider class of rules, called trading cycles rules, which characterize a class of Pareto-efficient and group strategy-proof rules. See Sönmez and Ünver (2011) for a recent survey.

A dynamic house allocation problem can be classified depending on how and when agents arrive and exit. With the deterministic arrival and exit of agents like ours, Bloch and Cantala (2008) independently consider a model similar to ours. Instead of rules as a function of preferences, they focus on a Markovian assignment rule as a function of the past assignment.

An important application of house allocation problems is kidney exchange for patients (Roth, Sönmez, and Ünver, 2004, 2005). Ünver (2010) studies a dynamic kidney exchange problem where agents arrive stochastically. We cannot apply our model to kidney exchange for two reasons. First, a patient with live donors (i.e. an agent with property rights) arrives in each period, whereas in our model only the initial existing tenants may have endowments. Second, kidney patients immediately leave the market once their exchange is done, but our model does not allow for this.

Let us turn to the model without the arrival and exit of agents. Abdulkadiroğlu and Loertscher (2007) study a dynamic house allocation problem with two periods where each agent's type is drawn in each period. Kamijo and Kawasaki (2010) study stability and competitive equilibrium in the dynamic housing market with time-invariant preferences. This type of model can be analyzed by static models: in multiple-type housing markets (Konishi, Quint, and Wako, 2001; Wako, 2005; Klaus, 2008), the number of types is interpreted as the life span of agents; in multiple assignment problems (Pápai, 2000b, 2001; Klaus and Miyagawa, 2001; Ehlers and Klaus, 2003), the quota of houses is identical across houses and then interpreted as the life span of agents. Adding the feasibility constraint of matching where a house is consumed by at most one agent in each period, we can see this as a dynamic model with time-invariant preferences.

Finally, there is a growing literature on dynamic rules with monetary transfers. For example, see Athey and Segal (2007) and Gershkov and Moldovanu (2009). 
Table 1: Demographic structure and a plan: The left table shows when an agent is present. For example, agent $a_{i}^{t}$ is present in periods $t$ and $t+1$. The right table shows a plan $\{\mu(t)\}_{t=1}^{\infty}$ where there are two houses $h_{1}$ and $h_{2}$, and only one agent arrives in each period.

\begin{tabular}{|c|c|c|c|c|c|c|}
\hline & 1 & 2 & $\cdots$ & $t$ & $t+1$ & $\cdots$ \\
\hline$a_{i}^{0}$ & - & & & & & \\
$a_{i}^{1}$ & - & - & & & & \\
$\vdots$ & & & $\ddots$ & & & \\
$a_{i}^{t-1}$ & & & & - & & \\
$a_{i}^{t}$ & & & & - & - & \\
$a_{i}^{t+1}$ & & & & & - & $\cdots$ \\
$\vdots$ & & & & & & $\ddots$ \\
\hline
\end{tabular}

\begin{tabular}{|c|c|c|c|c|c|c|}
\hline & 1 & 2 & $\cdots$ & $t$ & $t+1$ & $\cdots$ \\
\hline & $\mu(1)$ & $\mu(2)$ & $\cdots$ & $\mu(t)$ & $\mu(t+1)$ & $\cdots$ \\
\hline$a^{0}$ & $h_{1}$ & & & & & \\
$a^{1}$ & $h_{2}$ & $h_{1}$ & & & & \\
$\vdots$ & & & $\ddots$ & & & \\
$a^{t-1}$ & & & & $h_{1}$ & & \\
$a^{t}$ & & & & $h_{2}$ & $h_{2}$ & \\
$a^{t+1}$ & & & & & $h_{1}$ & $\cdots$ \\
$\vdots$ & & & & & & $\ddots$ \\
\hline
\end{tabular}

\section{The Model}

Time is discrete, starts at $t=1$, and lasts forever. There is a finite set, $H$, of indivisible goods, called houses, which are collectively owned. Houses are infinitely durable.

In each period $t, n$ agents, where $n \in \mathbb{N}_{++}$, arrive to consume houses for two periods, and then leave. In period 1 , there are $n$ initial existing tenants who live only in period 1 . We imagine that initial existing tenants have arrived in period 0 before the model starts. An agent is denoted by $a_{i}^{t}(t \geq 0$ and $i=1, \cdots, n)$ where the superscript indicates her arrival period and the subscript indexes her in her cohort (Table 1). Let $N(t):=\left\{a_{1}^{t}, \cdots, a_{n}^{t}\right\}$. In each period, the agents who have just arrived are called newcomers, while those who have arrived in the previous period are called existing tenants. In other words, in period $t$, newcomers are agents in $N(t)$, and existing tenants are agents in $N(t-1)$. Let $E(t):=N(t-1)$ be the set of all existing tenants in period $t$. In particular, $E(1)$ is the set of all initial existing tenants. Unlike the model of Abdulkadiroğlu and Sönmez (1999), here existing tenants do not necessary have property rights for a house. Let $A(t):=N(t) \cup E(t)$ be the set of all agents present in period $t$. Thus, for each period $t \geq 1$, $|A(t)|=2 n$. Let $A:=\cup_{t=1}^{\infty} A(t) \equiv E(1) \cup\left(\cup_{t=1}^{\infty} N(t)\right)$ be the set of all agents. Note that there is an infinite number of periods and also an infinite number of agents in this model. It is this "double infinity" that differentiates ours from the static models, which always assume a finite number of agents and houses. ${ }^{14}$

In each period, each agent consumes one house. The number of houses is equal to that of agents present in each period, that is, $|H|=|A(t)|=2 n$. We fix $H$ and $A$ throughout the paper.

Each initial existing tenant, $a^{0}$, has a strict preference relation, ${ }^{15} R_{a^{0}}$, on $H$. On the other hand, consider an agent, $a$, who is a newcomer in period $t \geq 1$. Her consumption set is $H \times H$.

\footnotetext{
${ }^{14}$ The double infinity is the major source of the theoretical peculiarities of the standard OLG model (Shell, 1971).

${ }^{15} \mathrm{~A}$ preference relation is a complete, reflexive, and transitive binary relation. A strict preference relation is a preference relation where no two distinct alternatives are indifferent.
} 
$\left(h_{t}, h_{t+1}\right) \in H \times H$ indicates that $h_{t}$ and $h_{t+1}$ are the consumed houses when she is a newcomer and an existing tenant, respectively. She has a (weak) preference relation $R_{a}$ on $H \times H$, which is not always strict. Let $P_{a}$ be the asymmetric part of $R_{a}$. Given $x, x^{\prime} \in H \times H, x R_{a} x^{\prime}$ means that agent $a$ finds $x$ at least as desirable as $x^{\prime}$, and $x P_{a} x^{\prime}$ means that she prefers $x$ to $x^{\prime}$. Moreover, the preference relation $R_{a}$ is time separable, ${ }^{16}$ that is, there are strict preferences $R_{a}(t)$ and $R_{a}(t+1)$ on $H$ such that for each $h_{t}, h_{t+1}, h_{t}^{\prime}, h_{t+1}^{\prime} \in H$,

1. if $h_{t} R_{a}(t) h_{t}^{\prime}$ and $h_{t+1} R_{a}(t+1) h_{t+1}^{\prime}$, then $\left(h_{t}, h_{t+1}\right) R_{a}\left(h_{t}^{\prime}, h_{t+1}^{\prime}\right)$,

2. if $h_{t} R_{a}(t) h_{t}^{\prime}$ and $h_{t+1} R_{a}(t+1) h_{t+1}^{\prime}$ such that at least one of them holds with $P_{a}(t)$ or $P_{a}(t+1)$, then $\left(h_{t}, h_{t+1}\right) P_{a}\left(h_{t}^{\prime}, h_{t+1}^{\prime}\right)$.

We call $R_{a}(t)$ a period $t$ preference. A preference $R_{a}$ is time invariant if all of its period preferences are identical, i.e., $R_{a}(t)=R_{a}(t+1)$. Note that if we know the preference relation $R_{a}$ of agent $a$ in $N(t)$, we obtain a collection $\left(R_{a}(t), R_{a}(t+1)\right)$ of her period preferences, but not vice versa.

In some cases, a time-invariant preference is derived from a cardinal utility function on $H$ as the sum of utilities across periods: for some utility function $u_{a}: H \rightarrow \mathbb{R}$, a preference $R_{a}$ is represented by the utility function $U_{a}\left(h_{t}, h_{t+1}\right):=u_{a}\left(h_{t}\right)+u_{a}\left(h_{t+1}\right)$. In other words, the preference depends on how many periods an agent consumes each house.

Let $\mathcal{R}_{a}$ be the set of all preference relations of agent $a$, and $\mathcal{R}_{a}(t)$ be the set of all periodpreference relations of agent $a$ where for each initial existing tenant $a$, we take $\mathcal{R}_{a}(1)=\mathcal{R}_{a}$. Let $\mathcal{R}:=\prod_{a \in A} \mathcal{R}_{a}, \mathcal{R}(t):=\prod_{a \in A(t)} \mathcal{R}_{a}(t), \mathcal{R}_{-a}:=\prod_{b \in A \backslash\{a\}} \mathcal{R}_{b}$, and $\mathcal{R}_{-a}(t):=\prod_{b \in A \backslash\{a\}} \mathcal{R}_{b}(t)$. The generic elements are denoted by $R, R(t)$ and $R_{-a}$, and $R_{-a}(t)$, respectively.

We call a collection of all time-separable preference profiles the general preference domain. On the other hand, the time-invariant preference domain is the collection of all preference profiles where each agent has a time-invariant preference relation. This domain is appropriate when the period is so short that all agents perceive their period preferences to be unchanging over time.

A period $t$ matching, $\mu(t)$, is a bijection from $A(t)$ to $H$ such that house $\mu_{a}(t)$ is the one assigned to agent $a$. Let $\mathcal{M}(t)$ be the set of all period $t$ matchings. See Table 1 for an example. A plan, $\mu:=\{\mu(t)\}_{t=1}^{\infty}$, is a collection of period $t$ matchings. Let $\mathcal{M}$ be the set of all plans. $\mu_{a}$ indicates the houses assigned to agent $a \in A$ under plan $\mu \in \mathcal{M}$ : If $a \in E(1), \mu_{a}=\mu_{a}(1)$, and if $a \in N(t)$ with $t \geq 1, \mu_{a}=\left(\mu_{a}(t), \mu_{a}(t+1)\right)$.

Given a preference relation $R_{a}$ of agent $a$, initially defined over the product of houses, we extend it to the set of plans in the following natural way: agent $a$ prefers plan $\mu$ to plan $\nu$ if and only if

\footnotetext{
${ }^{16}$ As we discussed in the Introduction, our assumption of time-separable preference violates the discounted utility (DU) model in two ways: Even if her preference is time invariant, an agent may prefer an improving path of houses over declining paths, which violates the DU model. If not, a period preference in some period may be affected by houses experienced in prior or future periods, which violates the independence assumption of the DU model. We do not go into details of experimental results on the validity of these assumptions. See Frederick, Lowenstein, and O'Donoghue (2002), especially sections 4.2.4 and 4.2.5, for further discussions.
} 
she prefers $\mu_{a}$ to $\nu_{a}$. Similarly, we extend a period preference to the set of period matchings.

An endowment profile indicates on which house each initial existing tenant has a property right only in period 1. Formally, it is a period 1 matching restricted to the set of initial existing tenants, $\left.e(1)\right|_{E(1)}$. Each agent except the initial existing tenants has no property right on any house when she arrives. We consider two kinds of house allocation problems with overlapping generations, or simply, dynamic problems, depending on the assumption of endowments: In a dynamic problem without endowments $(A, H, R)$, no initial existing tenants have property rights, while in a dynamic problem with endowments $\left(A, H, R,\left.e(1)\right|_{E(1)}\right)$, all initial existing tenants have property rights.

\section{Spot Rules}

\subsection{Definitions}

In a dynamic rule, each agent is asked to send a message, and then a plan is determined based on the message profile sent by all agents. Formally, a dynamic rule is a function from $\prod_{a \in A} S_{a}$ to $\mathcal{M}$ where $S_{a}$ is the message space of agent $a \in A$. A dynamic rule is direct if for each $a \in A, S_{a}=\mathcal{R}_{a}$. A spot rule is a dynamic rule such that if $a$ is an initial existing tenant, $S_{a}=\mathcal{R}_{a} \equiv \mathcal{R}_{a}(1)$, and if $a$ is a newcomer in period $t \geq 1, S_{a}=\mathcal{R}_{a}(t) \times \mathcal{R}_{a}(t+1)$. Note that any spot rule is not direct. We consider two kinds of spot rules: In a spot rule with property rights transfer, in each period, the houses consumed by newcomers become their properties that they can trade or continue to consume in the next period, while those consumed by existing tenants become vacant in the next period. A spot rule without property rights transfer has no transfer of property rights. A period $t$ (static and direct) rule is a function from $R(t)$ to $\mathcal{M}(t)$. Note that a spot rule can be constructed from all period rules.

A plan $\mu$ dynamically Pareto dominates a plan $\nu$ at $R \in \mathcal{R}$ if for each $a \in A, \mu_{a} R_{a} \nu_{a}$, and for some $a \in A, \mu_{a} P_{a} \nu_{a}$. A plan is dynamically Pareto efficient at $R \in \mathcal{R}$ if it is not dynamically Pareto dominated by any other plan at $R$, i.e., there is no other plan that all agents find at least as desirable and at least one agent prefers. Denote by $\boldsymbol{P}_{D}$ the mapping that associates with each $R \in \mathcal{R}$ the set of all dynamically Pareto-efficient plans at $R$. A period $t$ matching $\mu(t)$ is statically Pareto efficient at $R(t) \in \mathcal{R}(t)$ if there is no $\nu(t) \in \mathcal{M}(t)$ such that for each $a \in A(t)$, $\nu_{a}(t) R_{a}(t) \mu_{a}(t)$, and for some $a \in A(t), \nu_{a}(t) P_{a}(t) \mu_{a}(t) .{ }^{17}$ Denote by $\boldsymbol{P}_{S}^{t}$ the mapping that associates with each $R(t) \in \mathcal{R}(t)$ the set of all statically Pareto-efficient period $t$ matchings at $R(t)$.

We introduce the notion of acceptability:

Definition 1. In a dynamic problem without endowments, a plan $\mu$ is acceptable at $R \in \mathcal{R}$ if for each existing tenant $a$ in period $t \geq 2, \mu_{a}(t) R_{a}(t) \mu_{a}(t-1)$. For a dynamic problem with endowments, it is acceptable at $R \in \mathcal{R}$ if the above condition holds, and for each initial existing

\footnotetext{
${ }^{17}$ The example in the Introduction shows that a plan consisting of statically Pareto-efficient period-matchings is not dynamically Pareto efficient.
} 
tenant $a \in E(1), \mu_{a}(1) R_{a}(1) e_{a}(1)$. Denote by $\boldsymbol{A}$ the mapping that associates with each $R \in \mathcal{R}$ the set of all acceptable plans at $R$.

As discussed in the Introduction, an acceptable plan is desirable to students and universities in on-campus housing, and is necessary for apartment assignments because of the rental contracts.

A direct dynamic rule $\Gamma$ is dynamically Pareto efficient if for each $R \in \mathcal{R}, \Gamma(R) \in \boldsymbol{P}_{D}(R)$. Moreover, it is acceptable if for each $R \in \mathcal{R}, \Gamma(R) \in \boldsymbol{A}(R)$. A period $t$ rule $\pi$ is statically Pareto efficient if for each $R(t) \in \mathcal{R}(t), \pi(R(t)) \in \boldsymbol{P}_{S}^{t}(R(t))$.

To define properties for a spot rule, we introduce some notation: For each $a \in A$ and each $R_{a} \in \mathcal{R}_{a}$, denote by $p_{a}\left(R_{a}\right)$ a function that transforms her preference $R_{a}$ to her constituent period preferences. That is, if $a \in E(1), p_{a}\left(R_{a}\right)=R_{a}$, and if $a \in N(t)$ with $t \geq 1, p_{a}\left(R_{a}\right)=\left(R_{a}(t), R_{a}(t+\right.$ $1))$. Let $p(R):=\prod_{a \in A} p_{a}\left(R_{a}\right)$ and $p_{-a}\left(R_{-a}\right)=\prod_{b \in A \backslash\{a\}} p_{b}\left(R_{b}\right)$.

A spot rule $\Pi$ is dynamically Pareto efficient if for each $R \in \mathcal{R}, \Pi[p(R)] \in \boldsymbol{P}_{D}(R)$. Moreover, it is acceptable if for each $R \in \mathcal{R}, \Pi[p(R)] \in \boldsymbol{A}(R)$.

Proposition 1. If a spot rule is dynamically Pareto efficient (acceptable), then there is a direct dynamic rule that is dynamically Pareto efficient (acceptable).

Proof. For a spot rule $\Pi$, consider the direct dynamic rule $\Gamma$ : for each $R \in \mathcal{R}, \Gamma(R):=\Pi(p(R))$.

Let us now turn to incentive compatibility of rules. The definition of strategy-proofness in a direct (dynamic or period) rule requires truth-telling to be a dominant strategy for each agent, or equivalently, a profile of true preferences to be a Nash equilibrium in its associated preference revelation game. On the other hand, since a spot rule is not direct, we cannot use it for a spot rule. In a spot rule, an agent faces an extensive form with simultaneous moves. A strategy for an agent is her contingency plan. Note that truth-telling of her period preferences is a history-independent strategy that specifies only one period preference in each period. Our notion of incentive compatibility is as follows. ${ }^{18}$

Definition 2. A spot rule $\Pi$ is incentive compatible if a profile of true period preferences is a Nash equilibrium in its associated period-preference revelation game, i.e., for each $R \in \mathcal{R}$ and each $a \in A$,

- if $a$ is an initial existing tenant, then for each $\hat{R}_{a}(1) \in \mathcal{R}_{a}(1), \Pi[p(R)] R_{a} \Pi\left[\hat{R}_{a}(1), p_{-a}\left(R_{-a}\right)\right]$,

- if $a$ is a newcomer in period $t \geq 1$, then for each $\hat{R}_{a}(t) \in \mathcal{R}_{a}(t)$ and each $\hat{R}_{a}(t+1) \in \mathcal{R}_{a}(t+1)$, $\Pi[p(R)] R_{a} \Pi\left[\left(\hat{R}_{a}(t), \hat{R}_{a}(t+1)\right), p_{-a}\left(R_{-a}\right)\right]$.

\footnotetext{
${ }^{18}$ If our incentive compatibility required truthful revelation of period preferences to be a dominant strategy for each agent, our main positive results of Proposition 3 and Theorem 5 would not hold. But if a spot rule is incentive compatible as in Definition 2, then the truthful revelation is a dominant strategy among history-independent strategies for each agent, but not vice versa (this is because an agent can benefit from a strategy depending on history-dependent strategies of the others).

In our specific rules of SD and TTC, which are introduced in Sections 4 and 5 , if a profile of true period preferences is a Nash equilibrium, it is also a subgame perfect equilibrium. This is shown in footnotes 21 and 24.
} 
Our incentive compatibility of a spot rule is linked with strategy-proofness of a direct dynamic rule as follows:

Proposition 2 (Revelation principle). If a spot rule is incentive compatible, then there is a direct dynamic rule that is strategy-proof.

Proof. Given a spot rule $\Pi$, define the direct dynamic rule $\Gamma$ : for each $R \in \mathcal{R}, \Gamma(R):=\Pi(p(R))$. Let $R \in \mathcal{R}, a \in A$, and $R_{a}^{\prime} \in \mathcal{R}_{a}$. Since a spot rule $\Pi$ is incentive compatible, $\Pi[p(R)] R_{a}$ $\Pi\left[p_{a}\left(R_{a}^{\prime}\right), p_{-a}\left(R_{-a}\right)\right]$. Thus, $\Gamma(R) R_{a} \Gamma\left(R_{a}^{\prime}, R_{-a}\right)$.

In spite of the revelation principle, because the spot rule captures the situation where a static rule is applied in each period as in real-life markets, we focus on the spot rule.

\subsection{Impossibility result}

We might want to search for a dynamically Pareto-efficient and acceptable spot rule. The following result rules out such a rule. The proof is in the Appendix.

Theorem 1. ${ }^{19}$ Consider the general preference domain in a dynamic problem with or without endowments. Suppose that at least two agents arrive in each period. Then no spot rule is dynamically Pareto efficient and acceptable.

In checking dynamic Pareto efficiency, we have to look at two types of exchanges that do not exist in a static model: infinite exchanges between agents from different cohorts as in the example of the Introduction, and finite exchanges across periods among agents in the same cohort. The former is specific to the OLG structure. The latter, which we focus on in our proof, is for any kind of dynamic model.

In the proof, we assume that there is a spot rule satisfying dynamic Pareto efficiency and acceptability. We first specify a preference profile. Using the two properties, we narrow down a possible plan as a candidate of the plan induced by the spot rule in the following way. We pick two agents from the same cohort whose period preferences are reversed across two periods (thus, their preferences are not time invariant). Then, acceptability constrains the possible plan. For example, if an agent $a$ is assigned a house $h$ that is the worst house in her period preference $R_{a}(t)$ and the next period preference $R_{a}(t+1)$ ranks $h$ as her top choice, then by acceptability she must be assigned $h$ in the next period. Finally, we use the property of spot rules: when two preference profiles are distinct but have the same period-preference profile, by definition, a spot rule induces the same plan. Thus, we find another preference profile with the same period-preference profile where a candidate plan might be dynamically Pareto efficient in one preference profile, but not so in another profile. This contradicts the dynamic Pareto efficiency of the spot rule.

\footnotetext{
${ }^{19}$ If there are at least two agents who live for at least three periods, then we have the impossibility result for a direct dynamic rule: no direct dynamic rule is dynamically Pareto efficient and acceptable. See Kurino (2009) for the detail.
} 
Restricting our attention to the time-invariant preference domain, we show that we avoid the impossibility result for a dynamic problem both with and without endowments (Remarks 1 and 2).

\section{Serial Dictatorship (SD) Spot Rules}

We consider a specific spot rule without property rights transfer, called a serial dictatorship (SD) spot rule, whose underlying period rule is a serial dictatorship (SD) rule. For simplicity, we always consider the rule for a dynamic problem without endowments, so that no agent has property rights in any period.

\subsection{Period and cohort orderings of agents}

All spot rules that we examine are based on some ordering of agents. Given a set $B \subseteq A$ of agents, an ordering in $B$ is a linear ordering, ${ }^{20}$ denoted by $f_{B}$. We denote it as the ordered list:

$$
f_{B}:=\left(b_{1}, b_{2}, \cdots, b_{m}\right) \text { if and only if } b_{1} f_{B} b_{2} f_{B} \cdots f_{B} b_{m}
$$

We say that $b_{1}$ is the first agent in $B, b_{2}$ is the second agent in $B$, and so on. In addition, agent $a$ has a higher order than agent $b$ if $a f_{B} b$.

We look at two specific orderings: A period $t$ ordering is an ordering in $A(t)$ that is the set of all agents present in period $t$. A cohort ordering is an ordering of agents in the same cohort, i.e., an ordering in $E(1)$ or $N(t)$ for $t \geq 1$.

To investigate what kind of period orderings have an effect on various properties of spot rules, we elaborate on the period orderings:

Definition 3. 1. A period $t$ ordering $f_{A(t)}$ favors existing tenants if each existing tenant has a higher order than any newcomer in $f_{A(t)}$. It favors newcomers if each newcomer has a higher order than any existing tenant in $f_{A(t)}$.

2. A sequence of period orderings favors existing tenants (newcomers) if in each period $t$, the period $t$ ordering favors existing tenants (newcomers).

3. A sequence of period orderings is constant if the relative ranking of agents is the same across periods. That is, if an agent $a$ has a higher order than another agent $a^{\prime}$ in some period, then $a$ has a higher order than $a^{\prime}$ in any other period when they are both present.

In later sections, we investigate spot rules when the underlying sequence of period orderings is constant and favors existing tenants. In this case, we can create the ordering of all agents by which we can reproduce the period orderings:

\footnotetext{
${ }^{20} \mathrm{~A}$ linear ordering is a complete, reflexive, transitive, and antisymmetric binary relation.
} 
Observation 1. Given a constant sequence $\left\{f_{A(t)}\right\}_{t=1}^{\infty}$ of period ordering favoring existing tenants, we can construct an ordering in the set $A$ of all agents, denoted by $F$, which consists of cohort orderings $\left(f_{E(1)},\left\{f_{N(t)}\right\}_{t=1}^{\infty}\right)$ such that $F:=\left(f_{E(1)}, f_{N(1)}, f_{N(2)}, \cdots\right), f_{A(1)}=\left.\left(f_{E(1)}, f_{N(1)}\right) \equiv F\right|_{A(1)}$, and for each $t \geq 2, f_{A(t)}=\left(f_{N(t-1)}, f_{N(t)}\right)=\left.F\right|_{A(t)}$.

This observation is the key to prove our main positive results.

\subsection{Definition: SD spot rules}

In period $t \geq 1$, agents in $A(t)$ are present, houses in $H$ are available, each agent $a \in A(t)$ has a strict period preference $R_{a}(t)$ on $H$, and no agent has property rights on any house. That is, it is a house allocation problem $(A(t), H, R(t))$ (Hylland and Zeckhauser, 1979).

Let $R(t) \in \mathcal{R}(t)$, and $f_{A(t)}$ be a period $t$ ordering. A serial dictatorship (SD) period $t$ rule induced by $f_{A(t)}$ is determined as follows.

Step 1: The first agent in $f_{A(t)}$ is assigned her top choice according to $R_{a}(t)$. $\vdots$

Step $k$ : The $k^{t h}$ agent in $f_{A(t)}$ is assigned her top choice among the remaining houses according to $R_{a}(t)$.

It is known that an SD static rule is strategy-proof and statically Pareto efficient (Svensson, 1994).

Now, we are ready to define an SD spot rule: A serial dictatorship (SD) spot rule induced by a sequence of period orderings, $\left\{f_{A(t)}\right\}_{t=1}^{\infty}$, is a spot rule without property rights transfer where for each period $t$, the period $t$ rule is the SD period $t$ rule induced by $f_{A(t)}$. Moreover, an SD spot rule favoring existing tenants (newcomers) is an SD spot rule induced by some sequence of period orderings favoring existing tenants (newcomers). Also, a constant SD spot rule is an SD spot rule induced by some constant sequence of period orderings. When we say an SD spot rule, we mean an SD spot rule induced by some sequence of period orderings.

An SD spot rule consists of statically Pareto-efficient and strategy-proof SD period rules. The question is whether these properties can carry over to those in the dynamic setting. We see that the answer depends on whether it favors existing tenants or newcomers.

\subsection{Strategy-proofness}

An SD spot rule consists of strategy-proof period rules. Also, it ignores the past assignment in each period. Thus, we have:

Proposition 3. In the general preference domain, any SD spot rule is incentive compatible. ${ }^{21}$

\footnotetext{
${ }^{21}$ In an incentive-compatible SD spot rule without property rights transfer, a profile of true period preferences is a subgame perfect equilibrium: To see this, note that by definition, a profile of true period preferences is a Nash equilibrium. Also, since a spot rule has no property rights transfer, each history starts an SD spot rule for a new dynamic problem without endowments which is isormorphic to the original. This induced SD spot rule is incentive compatible, so a profile of true period preferences restricted to the game tree at the history is a Nash equilibrium.
} 
Proof. (Sketch) The logic is useful in understanding another spot rule in the next section. The point is that in any period an agent cannot affect the assignments of others with a higher order than her by lying. ${ }^{22}$

Consider any newcomer. When it is her turn to choose in the algorithm, all agents with a higher order than she are already assigned houses. Thus, her lying cannot influence the assignments of higer-order agents and get her a preferred house. On the other hand, consider any existing tenant. Since the past assignment is irrelevant to the current period rule, we can use the same logic to say that she cannot get a preferred house. By time-separability of preferences, she finds the assignment under true period-preferences at least as desirable as the one under false period-preferences.

\subsection{Dynamic Pareto efficiency and acceptability}

It is straightforward to obtain the following by generalizing the example in the Introduction to the case where there are at least two newcomers.

Theorem 2. Even in the time-invariant preference domain, an SD spot rule favoring newcomers is not dynamically Pareto efficient.

As in the example of the Introduction, in some preference profile, an SD spot rule favoring newcomers induces a plan that is dynamically Pareto dominated by the one from an infinite exchange between newcomers and existing tenants. In this case, there is a conflict among agents from different cohorts. ${ }^{23}$ As we saw in the impossibility result, another type of exchange-a finite exchange among those in the same cohort-can be a source of dynamic Pareto inefficiency. However, when an SD spot rule favors existing tenants and is constant, such possibilities are also excluded:

Theorem 3. In the general preference domain, a constant SD spot rule favoring existing tenants is dynamically Pareto efficient.

The proof is in the Appendix. Here is the intuition: By Observation 1, we can have the ordering $F$ of all agents such that we can reproduce each period $t$ ordering by restricting $F$ to $A(t)$. Thus, the first agent in $F$ is always the first in period orderings. Thus, she is assigned her most preferred house according to her period preference in each period. Hence, she cannot be better off. The second agent in $F$ is the second in period orderings unless the first agent leaves. If the first agent leaves, the second agent becomes the first in period orderings. Thus, the second agent is assigned her most preferred among the houses except the first agent's assigned house in each period. Hence, the second agent cannot be better off without hurting the first agent in $F$. Repeating this argument gives the desired result.

\footnotetext{
${ }^{22}$ This logic would fail if incentive compatibility were defined as a dominant strategy of truth-telling for each agent.

${ }^{23}$ We discuss a preference domain in the Conclusion where there is no such conflict. In this case, an SD spot rule favoring newcomers is dynamically Pareto efficient.
} 
Note that dynamic Pareto efficiency is achieved by the spot rule that extracts only period preferences, not the full preferences. Moreover, this spot rule becomes more desirable when agents have time-invariant preferences, as the following indicates.

Proposition 4. In the general preference domain, when at least two agents arrive in each period, a constant SD spot rule favoring existing tenants is not acceptable. However, in the time-invariant preference domain, it is acceptable.

Proof. By Theorem 3, such a spot rule is dynamically Pareto efficient. By the impossibility result, it is not acceptable. The proof of the second part is obvious.

Remark 1. In the time-invariant preference domain, by Theorem 3 and Proposition 4, we avoid the impossibility result, i.e., a constant SD spot rule favoring existing tenants is dynamically Pareto efficient and acceptable.

\section{$5 \quad$ Top Trading Cycles (TTC) Spot Rules}

In apartment assignments for an apartment management company, tenants cannot be evicted from the currently rented apartment. This constrains a spot rule to be acceptable. Moreover, acceptability is desirable to both students and universities for on-campus housing. In this section, we investigate an acceptable spot rule.

We consider a specific spot rule with property rights transfer, called a TTC spot rule, whose underlying static rule is Abdulkadiroğlu and Sönmez's top trading cycles (AS-TTC). For simplicity, we always consider the rule for a dynamic problem with endowments so that all existing tenants have property rights on houses.

\subsection{Definitions}

In period $t \geq 1$, agents in $A(t) \equiv E(t) \cup N(t)$ are present and houses in $H$ are available. Each agent $a$ has a period preference $R_{a}(t)$ on $H$. Each existing tenant in $E(t)$ has the property rights on the house she lived in the previous period, while newcomers in $N(t)$ do not. That is, it is a house allocation problem with existing tenants $\left(E(t), N(t),\left.e(t)\right|_{E(t)}, R(t), H\right)$ where each existing tenant $a$ has the property right on house $e_{a}(t)$ (Abdulkadiroğlu and Sönmez, 1999). To simplify the expression, when agent $a$ has the property rights on house $h$, we say that $a$ owns or occupies $h$, and $a$ is the owner of $h$.

A period $t$ matching $\mu(t)$ is individually rational at $R(t)$ if for each existing tenant $a \in E(t)$, $\mu_{a}(t) R_{a}(t) e_{a}(t)$, that is, she is assigned a house at least as desirable as her owned house.

Given an endowment profile $\left.e(t)\right|_{E(t)}$, a period $t$ static rule assigns a period $t$ matching for each period-preference profile. A period $t$ rule is individually rational if it always selects an individually rational period $t$ matching for each problem. 
Abdulkadiroğlu and Sönmez (1999) propose a rule referred to as Abdulkadiroğlu and Sönmez's top trading cycles (AS-TTC) period $t$ rule: Let $f_{A(t)}$ be a period $t$ ordering, $R(t) \in$ $\mathcal{R}(t)$, and an endowment profile, $\left.e(t)\right|_{E(t)}$. The rule selects a period $t$ matching through the following AS-TTC algorithm:

- Assign the first agent her top choice, the second agent her top choice among the remaining houses, and so on, until someone requests the house of an existing tenant.

- If at that point the existing tenant whose house is requested is already assigned another house, then do not disturb the procedure. Otherwise modify the remainder of the ordering by inserting the existing tenant before the requester at the ordering of agents and proceed with the first step of procedure through this existing tenant.

- Similarly, insert any existing tenant who has not already been served just before the requestor in the ordering of agents once her house is requested by an agent.

- If at any point a cycle forms, it is formed by exclusively existing tenants and each of them requests the house of the existing tenant who is next in the cycle. (A cycle is an ordered list of agents, $\left(a_{1}, a_{2}, \cdots, a_{k}\right)$, where agent $a_{1}$ demands the house of agent $a_{2}$, agent $a_{2}$ demands the house of agent $a_{3}, \cdots$, agent $a_{k}$ demands the house of $a_{1}$.) In such cases, remove all agents in the cycle by assigning them the houses they demand and proceed similarly.

Example 1 (The execution of AS-TTC algorithm). This example will be used in the proof of Theorem 6. We consider period $t=2$. Let $E(2)=\left\{a_{1}^{1}, a_{2}^{1}\right\}, N(2)=\left\{a_{1}^{2}, a_{2}^{2}\right\}$, and $H=$ $\left\{h_{1}, h_{2}, h_{3}, h_{4}\right\}$. Suppose $a_{1}^{1}$ owns $h_{3}$ and $a_{2}^{1}$ owns $h_{2}$. Let $R(2) \in \mathcal{R}(2)$ be given as:

\begin{tabular}{|c|c|c|c|}
\hline$a_{1}^{1}$ & $a_{2}^{1}$ & $a_{1}^{2}$ & $a_{2}^{2}$ \\
\hline$h_{1}$ & $h_{1}$ & $h_{2}$ & $h_{4}$ \\
$\frac{h_{3}}{h_{2}}$ & $\frac{h_{2}}{h_{3}}$ & & \\
\hline
\end{tabular}

Let $f_{A(2)}=\left(a_{1}^{2}, a_{2}^{2}, a_{1}^{1}, a_{2}^{1}\right)$ be the period 2 ordering that favors newcomers. The following four figures illustrates the execution of AS-TTC algorithm that proceeds from left to right. In each of the figures, the orderings of remaining agents are from left to right, and the owned houses $h_{2}$ and $h_{3}$ point to their owners $a_{2}^{1}$ and $a_{1}^{1}$ as long as the owners are not removed, while available houses do not point to any agent.
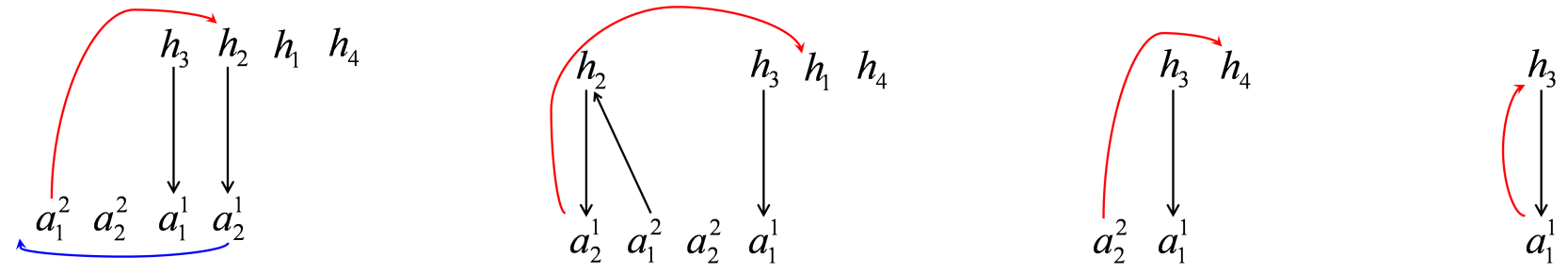
In the first graph, the first agent is $a_{1}^{2}$, who requests $h_{2}$ of existing tenant $a_{2}^{1}$. Because existing tenant $a_{2}^{1}$ is not assigned a house, modify the ordering by inserting existing tenant $a_{2}^{1}$ before the requester $a_{1}^{2}$, as indicated by the blue arrow; in the second graph, the first agent is $a_{2}^{1}$, who requests vacant house $h_{1}$. Thus, assign agent $a_{2}^{1}$ house $h_{1}$. Then, the second agent is $a_{1}^{2}$, who requests $h_{2}$ of existing tenant $a_{2}^{1}$. This time, since $a_{2}^{1}$ is already assigned, assign agent $a_{1}^{2}$ her requesting house $h_{2}$. Remove the matched agents and houses; in the third graph, the first agent is $a_{2}^{2}$, who requests vacant house $h_{4}$. Assign agent $a_{2}^{2}$ her requesting house $h_{4}$; in the last graph, the first agent is $a_{1}^{1}$, who requests house $h_{3}$ owned by herself. This forms a trivial cycle consisting only of $a_{1}^{1}$. Assign agent $a_{1}^{1}$ her owned house $h_{3}$. There are no agents left, and so the procedure stops. As a result, $\mu_{\left(a_{1}^{1}, a_{2}^{1}, a_{1}^{2}, a_{2}^{2}\right)}(2)=\left(h_{3}, h_{1}, h_{2}, h_{4}\right) . \diamond$

Note that any AS-TTC static rule is individually rational. This is because a house owned by an existing tenant is not assigned to another agent before this existing tenant is assigned a house. If another agent demands the house of this existing tenant, she is promoted to the top of the ordering. At the top of the ordering, if there is no house available better than her owned house, then the existing tenant demands her own house. At this point, a trivial cycle consisting of this agent forms. Then, she leaves and is assigned at worst her owned house.

Theorem 4 (Abdulkadiroglu and Sönmez, 1999). For any period ordering, $f_{A(t)}$, the induced ASTTC static rule is individually rational, statically Pareto efficient, and strategy-proof.

A top trading cycles (TTC) spot rule induced by a sequence of period orderings $\left\{f_{A(t)}\right\}_{t=1}^{\infty}$ is a spot rule with property rights transfer in which each period $t$, the period $t$ rule is the AS-TTC static rule induced by period $t$ ordering $f_{A(t)}$. In the same way as SD spot rules, we can define a TTC spot rule favoring existing tenants (newcomers), a constant TTC spot rule, and a TTC spot rule.

Since an AS-TTC static rule is individually rational, any TTC spot rule is acceptable. Therefore, from the impossibility result, in the general preference domain, any TTC spot rule is not dynamically Pareto efficient. We focus on the time-invariant preference domain, because this domain avoids the impossibility result (Remark 2) and has different implications from those of static models.

\subsection{Strategy-proofness: some positive results}

Recall that because it ignores the past assignment in each period, an SD spot rule is incentive compatible (Proposition 3). By contrast, a TTC spot rule guarantees each agent a house that is at least as desirable as the previously assigned house. This opens up the possibility of manipulation in which an agent obtains a worse house by lying, expecting to be upgraded in the ordering by being requested by some other agent in the next period. But a specific seniority-based TTC spot rule excludes such a possibility: 
Theorem 5. In the time-invariant preference domain, a constant TTC spot rule favoring existing tenants is incentive compatible. ${ }^{24}$

To prove the theorem, we introduce the notion of effective period ordering introduced by Sönmez and Ünver (2005). For each ordering $f_{A(t)}$, the AS-TTC algorithm assigns houses in one of two possible ways:

1. There is a sub-ordering $\left(a^{1}, \cdots, a^{k}\right)$ of agents where $a^{1}$ demands the house of $a^{2}, a^{2}$ demands the house of $a^{3}, \cdots$, agent $a^{k-1}$ demands house of $a^{k}$, and $a^{k}$ demands any available house. We call such a sub-ordering a serial-ordering $(S)$.

2. There is a sub-ordering $\left(a^{1}, \cdots, a^{k}\right)$ of existing tenants where $a^{1}$ receives $a^{k}$, s house, $a^{k}$ receives $a^{k-1}$ 's house, $\cdots, a^{2}$ receives $a^{1}$ 's house. We call such sub-ordering a loop-ordering $(L)$.

For a given ordering, $f_{A(t)}$, we construct the effective period ordering, denoted by $g_{A(t)}$, as follows: Apply the AS-TTC algorithm and order agents according to how their assignments are finalized. When there is a loop-ordering, order these agents as in the loop-ordering.

Note that a period matching induced by an AS-TTC algorithm yields the same one induced by an SD period rule with this effective ordering. Also note that the effective period ordering is endogenous, depending on preferences and the exogenous period ordering.

We now examine how effective period orderings behave under a constant sequence of period orderings favoring existing tenants. Let $R \in \mathcal{R}$ and $\left\{f_{A(t)}\right\}_{t=1}^{\infty}$ be such a sequence of period orderings. By Observation 1, let $F:=\left(f_{E(1)}, f_{N(1)}, f_{N(2)}, \cdots\right)$ be the ordering of all agents consisting of cohort orderings with $\left.F\right|_{A(t)}=f_{A(t)}$. For expositional simplicity, we use

$$
f_{N(t)}:=\left(a_{1}^{t}, a_{2}^{t}, \cdots, a_{n}^{t}\right)
$$

for each $t \geq 1$. Observe that in period 1 ,

$$
g_{A(1)}=(\overbrace{\underbrace{X, \cdots, X}_{\text {initial existing tenants }}}^{E(1)}, \overbrace{\underbrace{S}_{a_{1}^{1}}, \underbrace{S}_{a_{2}^{1}}, \cdots, \underbrace{S}_{a_{n}^{1}}}^{N(1)})=(\overbrace{X, \cdots, X}^{E(1)}, f_{N(1)}),
$$

where $X$ is either $S$ or $L$. Recall that $S$ stands for a serial-ordering and $L$ for a loop-ordering. That is, since newcomers do not own a house and the ordering $f_{A(1)}$ favors existing tenants, initial existing tenants are higher up than newcomers in the ordering. Moreover, because each newcomer owns no house, she demands an available house and forms a serial-ordering consisting of herself.

\footnotetext{
${ }^{24}$ In an incentive-compatible TTC spot rule with property rights transfer, a profile of true period preferences is a subgame perfect equilibrium: To see this, note that by definition, a profile of true period preferences is a Nash equilibrium. Also, since a spot rule has property rights transfer, each history starts a TTC spot rule for a new dynamic problem with endowments where the initial existing tenants are the existing tenants and the endowments are the houses on which the existing tenants have property rights, which is isormorphic to the original. This induced TTC spot rule is incentive compatible, so a profile of true period preferences restricted to the game tree at the history is a Nash equilibrium.
} 
Now, consider period 2. Note that existing tenants in period 2 are also newcomers in period 1 and the houses of existing tenants in period 1 become vacant in period 2 . The first agent in $f_{A(2)}$ is $a_{1}^{1}$. In period 1 , she prefers her assignment to those assigned to the other newcomers. Thus, since her assignment in period 1 becomes her endowment in period 2, she never demands the house owned by agent $a_{i}^{1}, i \geq 2$, but demands her own or an available house. Repeat this argument until there is no existing agent left. Then, the first agent among newcomers is $a_{1}^{2}$. Since all existing tenants left the market in the algorithm, all remaining houses are not owned by any agent. Thus, she demands an available house. Repeating this argument for all of the other newcomers, we obtain

$$
g_{A(2)}=(\overbrace{\underbrace{X}_{a_{1}^{1}}, \cdots, \underbrace{X}_{a_{n}^{1}}}^{E(2)=N(1)}, \overbrace{a_{a_{1}^{2}}^{S}, \cdots, \underbrace{S}_{a_{n}^{2}}}^{\overbrace{S(2)}^{S}})=\left(f_{N(1)}, f_{N(2)}\right) .
$$

Repeating this process, in period $t \geq 3$, we obtain

$$
g_{A(t)}=(\overbrace{\underbrace{X}_{a_{1}^{t-1}}, \cdots, \underbrace{X}_{a_{n}^{t-1}}}^{E(t)=N(t-1)}, \overbrace{\underbrace{S}_{a_{1}^{t}}, \cdots, \underbrace{S}_{a_{n}^{t}}}^{N(t)})=\left(f_{N(t-1)}, f_{N(t)}\right) .
$$

Notice the dynamics of the TTC spot rule: exchanges involve only one agent as in a serial or loop ordering after the initial existing tenants leave.

Proof. (Sketch) Observe that a sequence of effective period orderings is constant among all agents who are not initial existing tenants. Also, a plan induced by the TTC spot rule coincides with the $\mathrm{SD}$ spot rule induced by a sequence of effective orderings. By these observations, the logic of the proof is similar to that in Proposition 3 for an SD spot rule.

Consider any initial existing tenant. She lives there only in period 1. Thus, she faces the strategy-proof AS-TTC period rule. Thus, truth-telling is a dominant strategy for her.

Consider any newcomer in period $t \geq 1$. In each period when she is present, by the construction of effective period orderings, we conclude that her lying does not influence the effective period orderings of the higher-order agents. Thus, her lying does not change the assignment of these agents. Thus, when it is her turn to demand a house in the algorithm, the houses available given her truth-telling and lying are the same. So truth-telling gets her a house at least as desirable as the one under lying. By time-separability of preferences, her assignment under truth-telling is at least as desirable as the one under lying.

\subsection{How can a TTC spot rule be manipulated?}

Theorem 6. Consider the time-invariant preference domain. Suppose that at least two agents arrive in each period. Then, a TTC spot rule favoring newcomers is not incentive compatible. 
Proof. Suppose that agents $a_{1}^{t}$ and $a_{2}^{t}$ arrive in each period $t \geq 1$, and there are initial existing tenants $a_{1}^{0}$ and $a_{2}^{0}$. Fix a sequence of period orderings that favors newcomers. Without loss of generality, $a_{1}^{t}$ has a higher order than $a_{2}^{t}$ in each period $t$. Note that this sequence may not be constant, i.e., $a_{2}^{t}$ may have a higher order than $a_{1}^{t}$ in the period $t+1$ ordering. Let $R \in \mathcal{R}$ be such that true period preferences satisfy the left table (from best to worst):

\begin{tabular}{|c|c|c|c|c|c|}
\hline$a_{1}^{0}$ & $a_{2}^{0}$ & $a_{1}^{1}$ & $a_{2}^{1}$ & $a_{1}^{2}$ & $a_{2}^{2}$ \\
\hline$\underline{h_{1}}$ & $\underline{h_{4}}$ & $h_{1}$ & $h_{1}$ & $h_{2}$ & $h_{4}$ \\
& & $h_{3}$ & $h_{2}$ & & \\
& & $h_{2}$ & $h_{3}$ & & \\
\hline
\end{tabular}

\begin{tabular}{|l|}
$a_{1}^{1}$ \\
\hline$h_{1}$ \\
$h_{2}$ \\
$h_{3}$ \\
\hline
\end{tabular}

where the underlined houses are the endowment of the initial existing tenants. Moreover, agent $a_{1}^{1}$ 's preference satisfies

$$
\left(h_{2}, h_{1}\right) P_{a_{1}^{1}}\left(h_{3}, h_{3}\right) .
$$

We see that agent $a_{1}^{1}$ has an incentive to lie by reporting the period preference in the right table in periods 1 and 2: Looking at Figure 1, in period 1, when it is $a_{1}^{1}$ 's turn to choose, $h_{2}$ and $h_{3}$ are available. Truth-telling gives her $h_{3}$ and lying gives her $h_{2}$. Looking at Figure 2, under truth-telling, $h_{1}$ is assigned to $a_{2}^{1}$. Under lying, $a_{1}^{1}$ successfully gets her top choice $h_{1}$. Overall, $a_{1}^{1}$ is assigned $\left(h_{3}, h_{3}\right)$ under truth-telling and is assigned $\left(h_{2}, h_{1}\right)$ under lying. Hence, she has an incentive to lie.

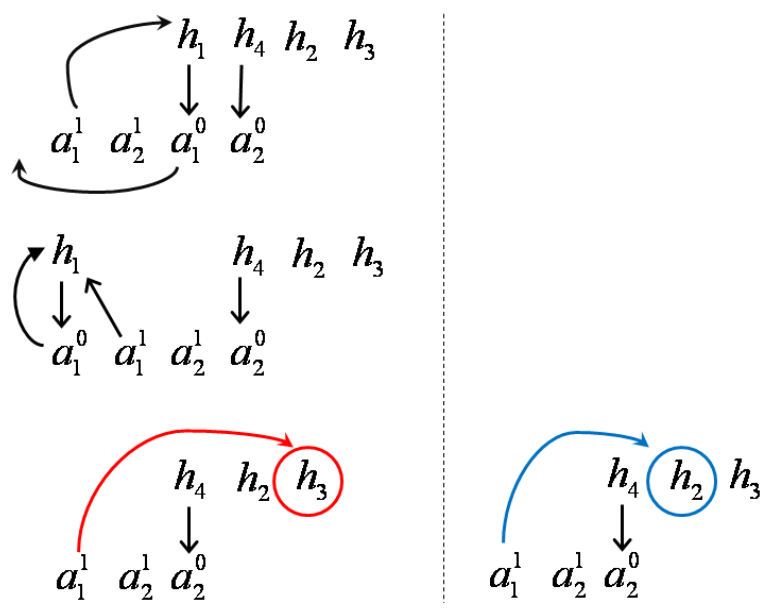

Figure 1: AS-TTC algorithms up to agent $a_{1}^{1}$ s s turn in period $t=1$ under the truthful preference (left) and the manipulated preference (right) in Theorem 6. 

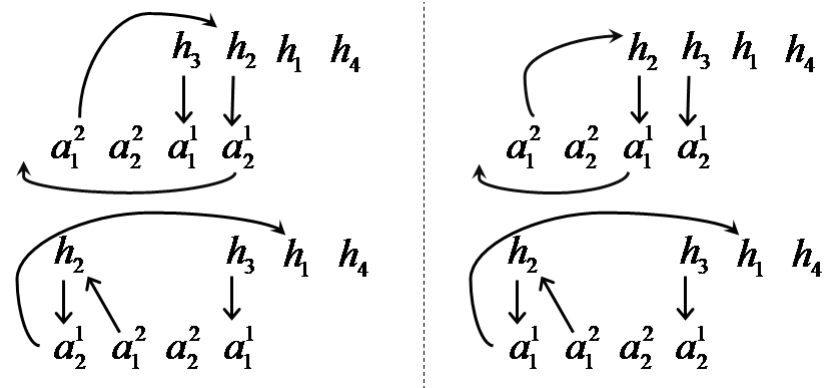

Figure 2: AS-TTC algorithms until $h_{1}$ is assigned in period $t=2$ under the truthful preference (left) and the manipulated preference (right). Here $a_{1}^{1}$ is the first and $a_{2}^{1}$ is the second, but the reverse ordering gives the same period matching in Theorem 6.

\subsection{Dynamic Pareto efficiency: some positive results}

Using the analysis of effective period orderings for a constant TTC spot rule favoring existing tenants, we obtain the following positive result.

Theorem 7. In the time-invariant preference domain, a constant TTC spot rule favoring existing tenants is dynamically Pareto efficient.

Proof. (Sketch) The proof is similar to that in Theorem 3 for the dynamic Pareto efficiency of a constant SD spot rule favoring existing tenants in that the sequence of effective period orderings is constant among all agents except initial existing tenants, and in each period the period matching induced by the AS-TTC rule yields the same period matching induced by the SD static rule with the effective period ordering. Thus, we need to take care of initial existing tenants. They face the Pareto-efficient AS-TTC period rule in period 1. Hence, they cannot be better off without hurting others.

Remark 2. Any TTC spot rule is acceptable. Thus, in the time-invariant preference domain, by Theorem 7, we avoid the impossibility result, i.e., a constant TTC spot rule favoring existing tenants is dynamically Pareto efficient and acceptable.

\subsection{When is a TTC spot rule undesirable?}

The example in the Introduction shows the dynamic Pareto inefficiency of an SD spot rule favoring newcomers. There, we demonstrate that an infinite exchange between existing tenants and newcomers dynamically Pareto dominates the plan induced by the SD spot rule favoring newcomers. Looking at this example more closely, we might think that acceptability precludes such an exchange. Since a TTC spot rule is acceptable, one might conjecture that a TTC spot rule favoring newcomers is dynamically Pareto efficient. However, this is not the case: 
Theorem 8. Consider the time-invariant preference domain. Suppose that at least two agents arrive in each period. Then, a TTC spot rule favoring newcomers is not dynamically Pareto efficient.

Proof. Suppose that agents $a_{1}^{t}$ and $a_{2}^{t}$ arrive in each period $t$, and there are initial existing tenants $a_{1}^{0}$ and $a_{2}^{0}$. Fix a sequence of period orderings that favors newcomers. Without loss of generality, we may assume $a_{1}^{t}$ is the first agent in each period $t$ ordering, and $a_{1}^{0}$ has a higher order than $a_{2}^{0}$. Note that this sequence may not be constant; $a_{2}^{t}$ may have a higher order than $a_{1}^{t}$ in the period $t+1$ ordering. But this does not affect the induced plan in the example below. Let preferences satisfy

\begin{tabular}{|c|c|c|c|c|c|c|c|c|c|}
\hline$a_{1}^{0}$ & $a_{2}^{0}$ & $a_{1}^{1}$ & $a_{2}^{1}$ & $a_{1}^{2}$ & $a_{2}^{2}$ & $a_{1}^{3}$ & $a_{2}^{3}$ & $a_{1}^{4}$ & $a_{2}^{4}$ \\
\hline$h_{3}$ & $h_{4}$ & $h_{4}$ & $h_{4}$ & $h_{3}$ & $h_{3}$ & $h_{1}$ & $h_{1}$ & $h_{2}$ & $h_{2}$ \\
$\underline{h_{1}}$ & $\underline{h_{2}}$ & & $h_{2}$ & & $h_{4}$ & & $h_{3}$ & & $h_{1}$ \\
& & & $h_{1}$ & & $h_{2}$ & & $h_{4}$ & & $h_{3}$ \\
& & & $h_{3}$ & & $h_{1}$ & & $h_{2}$ & & $h_{4}$ \\
\hline
\end{tabular}

\begin{tabular}{|c|c|c|c|}
\hline$a_{2}^{1}$ & $a_{2}^{2}$ & $a_{2}^{3}$ & $a_{2}^{4}$ \\
\hline$\left(h_{3}, h_{2}\right)$ & $\left(h_{1}, h_{4}\right)$ & $\left(h_{2}, h_{3}\right)$ & $\left(h_{4}, h_{1}\right)$ \\
$\left(h_{1}, h_{1}\right)$ & $\left(h_{2}, h_{2}\right)$ & $\left(h_{4}, h_{4}\right)$ & $\left(h_{3}, h_{3}\right)$ \\
\hline
\end{tabular}

The left table shows period preferences. The right table shows entire preferences. The houses owned by initial existing tenants are underlined in the left table. Each of the other agents has the following preferences: for each $t \geq 1$ and each $i=1,2$,

- Agent $a_{i}^{4 t+1}$ has the same preference as $a_{i}^{1}$ does,

- Agent $a_{i}^{4 t+2}$ has the same preference as $a_{i}^{2}$ does,

- Agent $a_{i}^{4 t+3}$ has the same preference as $a_{i}^{3}$ does, and

- Agent $a_{i}^{4 t+4}$ has the same preference as $a_{i}^{4}$ does.

The TTC spot rule induces the plan $\mu$ in Table 2. Clearly, $\mu$ is not dynamically Pareto efficient. Notice that starting with $\mu$, an infinite exchange of houses between agents $a_{2}^{t-1}$ and $a_{2}^{t}$ in each period $t(t \geq 2)$, dynamically Pareto dominates $\mu$.

\section{An Extension}

We study the general case where agents live for at least three periods. In this case, we show in Proposition 5 that a constant TTC spot rule favoring existing tenants is no longer incentive compatible nor dynamically Pareto efficient. The reason is that some of the initial existing tenants, who have property rights on houses in period 1, live for multiple periods. By contrast, any positive results of SD spot rules still hold, because initial existing tenants do not have property rights.

To highlight the above issue without notational complexity, we focus on three periods during which agents live. The case for any other span of periods can be extended in a straightforward way.

The model starts at $t=1$. In each period $t \geq 1, n$ agents arrive to live for three periods and then leave (Table 6). In period 1, there are two types of initial existing tenants: those who 
Table 2: In each cell, houses on the left side are the plan $\mu$ induced by the TTC spot rule, while houses with parentheses on the right are given by another plan in Theorem 8 .

\begin{tabular}{|l|l|l|l|l|l|l|l|}
\hline & $t=1$ & $t=2$ & $t=3$ & $t=4$ & $t=5$ & $t=6$ & $\cdots$ \\
\hline$a_{1}^{0}$ & $h_{3}$ & & & & & & \\
$a_{2}^{0}$ & $h_{2}$ & & & & & & \\
\hline$a_{1}^{1}$ & $h_{4}$ & $h_{4}$ & & & & & \\
$a_{2}^{1}$ & $h_{1}$ & $h_{1}\left(h_{2}\right)$ & & & & & \\
\hline$a_{1}^{2}$ & & $h_{3}$ & $h_{3}$ & & & & \\
$a_{2}^{2}$ & & $h_{2}\left(h_{1}\right)$ & $h_{2}\left(h_{4}\right)$ & & & & \\
\hline$a_{1}^{3}$ & & & $h_{1}$ & $h_{1}$ & & & \\
$a_{2}^{3}$ & & & $h_{4}\left(h_{2}\right)$ & $h_{4}\left(h_{3}\right)$ & & & \\
\hline$a_{1}^{4}$ & & & & $h_{2}$ & $h_{2}$ & & \\
$a_{2}^{4}$ & & & & $h_{3}\left(h_{4}\right)$ & $h_{3}\left(h_{1}\right)$ & & \\
\hline$a_{1}^{5}$ & & & & & $h_{4}$ & $h_{4}$ & \\
$a_{2}^{5}$ & & & & & $h_{1}\left(h_{3}\right)$ & $h_{1}\left(h_{2}\right)$ & \\
\hline$\vdots$ & & & & & & $\vdots$ & $\ddots$ \\
\hline
\end{tabular}

live in period 1 , denoted by $a_{i}^{-1}, i=1, \cdots, n$; and those who live in periods 1 and 2 , denoted by $a_{i}^{-1}, i=1, \cdots, n$. With a slight abuse of notation and for simplicity, we imagine that agent $a_{i}^{-1}$ has arrived in period -1 that is two periods before the model starts, and also $a_{i}^{0}$ has arrived in period 0 . For $t \geq-1$, let $N(t)=\left\{a_{1}^{t}, \cdots, a_{n}^{t}\right\}$ be the set of agents who arrive in period $t$. In each period $t \geq 1$, agents are from three different cohorts of $N(t-2), N(t-1)$, and $N(t)$. Agents in $E(t):=N(t-2) \cup N(t-1)$ are called existing tenants in period $t$, while agents in $N(t)$ are called newcomers in period $t$. Let $A(t):=E(t) \cup N(t)$ be the set of all agents present in period $t$. Also, let $A:=\cup_{t \geq 1} A(t)$ be the set of all agents. Preferences are defined in a similar way as in Section 2 . In a dynamic problem without endowments, no initial existing tenants have property rights. In a dynamic problem with endowments, all initial existing tenants in $E(1)$ have property rights on houses for period 1.

It is straightforward to extend all definitions and propositions of spot rules in Section 3, all definitions of period orderings and SD spot rules in Sections 4.1 and 4.2, all results of SD spot rules in Sections 4.3 and 4.4, and all definitions of TTC spot rules in Section 5.1. However, we cannot directly extend the results of TTC spot rules in Section 5, as the following shows.

Proposition 5. Consider the time-invariant preference domain. Suppose that agents live for three periods. Then, a constant TTC spot rule favoring existing tenants can be manipulated by an initial existing tenant if at least three newcomers arrive in each period, and is not dynamically Pareto efficient if at least two newcomers arrive in each period.

The proof is in the Appendix. The reason is that some of the initial existing tenants stay in the market for multiple periods. This can be seen by looking at the behavior of the effective period 
Table 3: Demographic structure when the life span is three: The table shows when an agent is present. For example, agent $a_{i}^{t}$ is present in periods $t, t+1$, and $t+2$.

\begin{tabular}{|c|c|c|c|c|c|c|c|c|}
\hline & 1 & 2 & 3 & $\cdots$ & $t$ & $t+1$ & $t+2$ & $\cdots$ \\
\hline$a_{i}^{-1}$ & - & & & & & & & \\
$a_{i}^{0}$ & - & - & & & & & & \\
$a_{i}^{1}$ & - & - & - & & & & & \\
$\vdots$ & & & & & & & & \\
$a_{i}^{t-2}$ & & & & & - & & & \\
$a_{i}^{t-1}$ & & & & & - & - & & \\
$a_{i}^{t}$ & & & & & - & - & - & \\
\hline
\end{tabular}

orderings. By the logic used in Section 5.2, we can obtain the following effective period orderings:

$$
g_{A(t)}= \begin{cases}(\overbrace{X, \cdots, X}^{E(1) \equiv N(-1) \cup N(0)}, f_{N(1)}) & \text { if } t=1, \\ (\overbrace{X, \cdots, X}^{E(1) \cap A(2)=N(0)}, f_{N(1)}, f_{N(2)}) & \text { if } t=2, \\ \left(f_{N(t-2)}, f_{N(t-1)}, f_{N(t)}\right) & \text { if } t \geq 3 .\end{cases}
$$

where $X$ is a serial-ordering or a loop-ordering. Observe that the above sequence of effective period orderings is constant among all agents except initial existing tenants. Thus, we weaken incentive compatibility and dynamic Pareto efficiency by focusing on all agents except initial existing tenants.

Definition 4. 1. A spot rule is incentive compatible among all agents except initial existing tenants ${ }^{25}$ if we drop condition (1) in Definition 2, i.e., the truthful revelation of period orderings is the best strategy for each agent who is not an initial existing tenant, given that all of the other agents reveal truthful period preferences.

(a) A plan $\nu$ dynamically Pareto dominates another plan $\mu$ among all agents except initial existing tenants at a preference profile $R$ if

$i$. for each $t \geq 1,\left\{\mu_{a}(t): a \in A \backslash E(1)\right\}=\left\{\nu_{a}(t): a \in A \backslash E(1)\right\}$, and

ii. for each $a \in A \backslash E(1), \nu R_{a} \mu$ and for some $a \in A \backslash E(1), \nu P_{a} \mu$.

(b) A plan is dynamically Pareto efficient among all agents except initial existing tenants at a preference profile $R$ if it is not dynamically Pareto dominated by any other plan among all agents except initial existing tenants at $R$.

(c) A spot rule is dynamically Pareto efficient among all agents except initial existing tenants if, for each preference profile, it always selects a plan that is dynamically Pareto efficient among all agents except initial existing tenants at this preference profile.

\footnotetext{
${ }^{25}$ Note that if a spot rule is incentive compatible among all agents except initial existing tenants, then truth-telling is a dominant strategy among history-independent strategies for each agent who is not an initial existing tenant.
} 
Then, we can restore the positive results while retaining the negative results:

Proposition 6. In the time-invariant preference domain, when agents live for three periods,

1. a constant TTC spot rule favoring existing tenants is incentive compatible and dynamically Pareto efficient among all agents except initial existing tenants, and

2. if at least two newcomers arrive in each period, then a TTC spot rule favoring newcomers is neither incentive compatible nor dynamically Pareto efficient among all agents except initial existing tenants.

Proof. For the first part, because the sequence of effective period ordering is constant among all agents except initial existing tenants, we can use the same logic employed in Theorems 5 and 7 . For the second part, it is straightforward to generalize the examples in Theorems 6 and 8 .

A constant TTC spot rule favoring existing tenants does not perform well when initial existing tenants are present, but it does perform well after they leave. This shows that in terms of market design, it is important to consider how to treat initial existing tenants and their property rights. It might be possible to invalidate the property rights of initial existing tenants to obtain full dynamic Pareto efficiency and incentive compatibility. If not, such a possibility is excluded but the TTC spot rule still performs well in the long run after initial existing tenants leave.

\section{Conclusion}

Real-life examples use the priority ordering of agents to allocate indivisible objects to agents. The ordering often favors seniors. As long as we stick to the static models, our explanation for this would be that seniors demand respect. However, turning to the dynamic structure inherent in real-life markets, this paper uncovers an important advantage of using seniority-based assignment orderings in terms of positive properties such as Pareto efficiency and incentive compatibility.

Although we have examined two preference domains, we can apply the rationale and the tools developed in this paper to the other kinds of domains. For example, suppose agents' preferences are known to evolve in a specific pattern: Suppose the number of houses is equally divided between quiet and noisy houses. Also, suppose agents live for two periods. Newcomers prefer noisy houses to quiet ones; existing tenants prefer the quiet to the noisy. Agents have heterogeneous period preferences for the same type of houses. Note that there is no conflict among different cohorts, that is, newcomers prefer noisy houses and existing tenants prefer quiet ones. Thus, if we apply a statically Paretoefficient rule as in a spot rule of SD and TTC, then newcomers are assigned the noisy and existing tenants are the quiet. Thus, each period rule is not affected by the previous assignment. Hence, an SD spot rule is acceptable and dynamically Pareto efficient as well as incentive compatible. Also, a TTC spot rule is incentive compatible and dynamically Pareto efficient as well as acceptable. 
The research into dynamic house allocation problems has started only recently. This paper offers a new perspective on the performance of seniority-based static rules. Our results raise several interesting questions about market design: Comparing the results of the two spot rules, we notice that an SD spot rule is robust to the dynamic structure, but a TTC spot rule is vulnerable to it. However, the property of acceptability, which is desirable and sometimes necessary in reallife markets, is not generally satisfied in an SD spot rule unlike a TTC spot rule. This might beg the question as to why AS-TTC rules have never been used, although the RSD rule with squatting rights is widely used despite the lack of some desirable static properties. Our model is limited and abstracts from many realistic situations in order to highlight the importance of seniority-based rules. Thus, it would be interesting to see how a TTC spot rule behaves under more realistic assumptions on the evolution of preferences based on experiences, the entry and exit of agents, and the introduction of an outside option. Finally, it would be interesting to study lottery rules in addition to the deterministic rules studied in this paper. These issues remain for future investigation.

\section{Appendix}

Proof of Theorem 1. Suppose that two agents arrive in each period, and live for two periods. Then, let $H:=\left\{h_{1}, \cdots, h_{4}\right\}$. Seeking a contradiction, suppose that there is such a spot rule $\Pi$. Pick two newcomers $a_{1}^{1}$ and $a_{2}^{1}$ in period 1 . Let $R \in \mathcal{R}$ such that

\begin{tabular}{|c|c|c|c|}
\hline \multicolumn{2}{|c|}{$a_{1}^{1}$} & \multicolumn{2}{c|}{$a_{2}^{1}$} \\
\hline$R_{a}(1)$ & $R_{a}(2)$ & $R_{a}(1)$ & $R_{a}(2)$ \\
\hline$h_{1}$ & $h_{2}$ & $h_{1}$ & $h_{2}$ \\
$h_{2}$ & $h_{1}$ & $h_{2}$ & $h_{1}$ \\
\hline
\end{tabular}

\begin{tabular}{|c|c|}
\hline$a_{1}^{1}$ & $a_{2}^{1}$ \\
\hline$R_{a}$ & $R_{a}$ \\
\hline$\left(h_{1}, h_{1}\right)$ & $\left(h_{2}, h_{2}\right)$ \\
$\left(h_{2}, h_{2}\right)$ & $\left(h_{1}, h_{1}\right)$ \\
\hline
\end{tabular}

Also, for each $a \in A \backslash\left\{a_{1}^{1}, a_{2}^{1}\right\}$, each $\tau \in \mathbb{N}$ when $a$ is present in $\tau$, and each $h \in H \backslash\left\{h_{1}, h_{2}\right\}$, $h R_{a}(\tau) h_{1}$ and $h R_{a}(\tau) h_{2}$.

Let $\mu:=\Pi[p(R)]$. Since $\mu \in \boldsymbol{P}_{D}(R)$, then for each $\tau=1,2, \mu(\tau) \in \boldsymbol{P}_{S}^{\tau}(R(\tau))$. Thus, for each $\tau=1,2$, either $\mu_{a_{1}^{1}, a_{2}^{1}}(\tau)=\left(h_{2}, h_{1}\right)$ or $\mu_{a_{1}^{1}, a_{2}^{1}}(\tau)=\left(h_{1}, h_{2}\right)$.

Case 1: $\mu_{a_{1}^{1}, a_{2}^{1}}(1)=\left(h_{2}, h_{1}\right)$. By acceptability, $\mu_{a_{1}^{1}}(2)=h_{2}$. Thus, $\mu_{a_{2}^{1}}(2)=h_{1}$. Thus, $\mu_{a_{1}^{1}}=\left(h_{2}, h_{2}\right)$ and $\mu_{a_{2}^{1}}=\left(h_{1}, h_{1}\right)$. Let $\nu \in \mathcal{M}$ such that $\nu_{a_{1}^{1}}=\left(h_{1}, h_{1}\right), \nu_{a_{2}^{1}}=\left(h_{2}, h_{2}\right)$, and for each $a \in A \backslash\left\{a_{1}^{1}, a_{2}^{1}\right\}$, $\nu_{a}=\mu_{a}$. Then, $\nu$ dynamically Pareto dominates $\mu$ at $R$. This contradicts $\mu \in \boldsymbol{P}_{\boldsymbol{D}}(R)$.

Case 2: $\mu_{a_{1}^{1}, a_{2}^{1}}(\tau)=\left(h_{1}, h_{2}\right)$. By the same reasoning as in Case 1, $\mu_{a_{1}^{1}}=\left(h_{1}, h_{1}\right)$ and $\mu_{a_{2}^{1}}=\left(h_{2}, h_{2}\right)$. Now, let $R^{\prime} \in \mathcal{R} \backslash\{R\}$ be such that for each $a \in A$ and each $t \in \mathbb{N}, R_{a}^{\prime}(t)=R_{a}(t)$, and

\begin{tabular}{|c|c|}
\hline$R_{a_{1}^{1}}$ & $R_{a_{2}^{1}}$ \\
\hline$\left(h_{2}, h_{2}\right)$ & $\left(h_{1}, h_{1}\right)$ \\
$\left(h_{1}, h_{1}\right)$ & $\left(h_{2}, h_{2}\right)$ \\
\hline
\end{tabular}

Then, $\Pi\left[p\left(R^{\prime}\right)\right]=\mu$. Let $\nu \in \mathcal{M}$ such that $\nu_{a_{1}^{1}}=\left(h_{2}, h_{2}\right), \nu_{a_{2}^{1}}=\left(h_{1}, h_{1}\right)$, and for each $a \in$ $A \backslash\left\{a_{1}^{1}, a_{2}^{1}\right\}, \nu_{a}=\mu_{a}$. Then, $\nu$ dynamically Pareto dominates $\mu$ at $R^{\prime}$. This contradicts $\mu \in \boldsymbol{P}_{\boldsymbol{D}}\left(R^{\prime}\right)$. 
Proof of Theorem 3. Let $R \in \mathcal{R}$. Let $f=\left\{f_{A(t)}\right\}_{t=1}^{\infty}$ be a constant sequence of period orderings that favors existing tenants. Let $\mu=S D^{f}(p(R))$. To find a contradiction, suppose that some plan $\nu$ dynamically Pareto dominates $\mu$ at $R$, i.e.,

$$
\text { for each } a \in A, \nu R_{a} \mu \text {, and for some } b \in A, \nu P_{b} \mu \text {. }
$$

From Observation 1, there is an ordering of all agents, $F=\left(f_{E(1)}, f_{N(1)}, f_{N(2)} \cdots\right)$ with $\left.F\right|_{A(t)}=$ $f_{A(t)}$. For convenience, suppose $F=\left(a_{1}, a_{2}, \cdots, a_{m}, \cdots\right)$. We show by induction on $m$ that for each $m=1,2, \cdots, \mu_{a_{m}}=\nu_{a_{m}}$, i.e., $\nu=\mu$, which contradicts (1).

Induction basis: $m=1$. Since $f$ favors existing tenants, $a_{1}$ is an initial existing tenant. Since $\left.F\right|_{A(1)}=f_{A(1)}$, she is the first agent in $f_{A(1)}$. Thus, she is assigned her most preferred house in $H$ according to $R_{a_{1}} \equiv R_{a_{1}}(1)$. Thus, $\mu_{a_{1}} R_{a_{1}} \nu_{a_{1}}$. By (1) and the strict preference of $R_{a_{1}}(1)$, we have $\mu_{a_{1}}=\nu_{a_{1}}$.

Induction step: Suppose that the claim is true up to $m-1$, i.e., $\mu_{a_{1}}=\nu_{a_{1}}, \cdots, \mu_{a_{m-1}}=\nu_{a_{m-1}}$. First, we show that

for each $t \in \mathbb{N}$ such that $a_{m}$ is present in period $t, \mu_{a_{m}}(t) R_{a_{m}}(t) \nu_{a_{m}}(t)$.

Let $t \in \mathbb{N}$ be such that agent $a_{m}$ is present in period $t$. Since $\left.F\right|_{A(t)}=f_{A(t)}$, then each agent $a \in A$ with a higher order than $a_{m}$ in $f_{A(t)}$ is one of agents $a_{1}, \cdots, a_{m-1}$. Thus, by the induction hypothesis, $\mu_{a}(t)=\nu_{a}(t)$. Thus, when it is $a_{m}$ 's turn to choose, the remaining houses are in $H \backslash\left\{\mu_{a}(t) \mid a=a_{1}, \cdots, a_{m-1}\right\}=H \backslash\left\{\nu_{a}(t) \mid a=a_{1}, \cdots, a_{m-1}\right\}$. Since $\mu_{a_{m}}(t)$ is $a_{m}$ 's most preferred house among the remaining houses, $\mu_{a_{m}}(t) R_{a_{m}}(t) \nu_{a_{m}}(t)$. Thus, (2) is true.

If $a_{m}$ is an initial existing tenant, since $R_{a_{m}}=R_{a_{m}}(1)$ is a strict preference, by (1) and (2), $\mu_{a_{m}}=\nu_{a_{m}}$. Suppose $a_{m}$ is a newcomer in period $t$. Then, by (2) and the first condition of timeseparability of preferences, $\mu_{a_{m}} R_{a_{m}} \nu_{a_{m}}$. Thus, by (1), $\nu_{a_{m}} R_{a_{m}} \mu_{a_{m}}$. Thus, from the second condition of time-separability, either [for some $\tau=t, t+1, \nu_{a_{m}}(\tau) P_{a_{m}}(\tau) \mu_{a_{m}}(\tau)$ ] or [for each $\left.\tau=t, t+1, \nu_{a_{m}}(\tau) R_{a_{m}}(\tau) \mu_{a_{m}}(\tau)\right]$. The former is impossible. Thus, for each $\tau=t, t+1$, $\nu_{a_{m}}(\tau) I_{a_{m}}(\tau) \mu_{a_{m}}(\tau)$ where $I_{a_{m}}(\tau)$ is the indifference relation of $R_{a_{m}}(\tau)$. Because the period preference is strict, for each $\tau=t, t+1, \nu_{a_{m}}(\tau)=\mu_{a_{m}}(\tau)$. That is, $\nu_{a_{m}}=\mu_{a_{m}}$.

Proof of Proposition 5. Suppose that agents live for three periods.

For the first part, suppose that three agents arrive in each period. We focus on initial existing tenants in $E(1)=\left\{a_{1}^{-1}, a_{2}^{-1}, a_{3}^{-1}, a_{1}^{0}, a_{2}^{0}, a_{3}^{0}\right\}$. Since there are six initial existing tenants, we explicitly consider six houses, $h_{1}$ through $h_{6}$. Period preferences of each initial existing tenant satisfy the left table below:

\begin{tabular}{|c|c|c|c|c|c|}
\hline$a_{1}^{-1}$ & $a_{2}^{-1}$ & $a_{3}^{-1}$ & $a_{1}^{0}$ & $a_{2}^{0}$ & $a_{3}^{0}$ \\
\hline$h_{1}$ & $h_{5}$ & $h_{3}$ & $h_{6}$ & $h_{1}$ & $h_{1}$ \\
& & & $h_{4}$ & $h_{2}$ & $h_{2}$ \\
& & & & & $h_{6}$ \\
$h_{6}$ \\
\hline
\end{tabular}


In addition, agent $a_{2}^{0}$ prefers $\left(h_{6}, h_{1}\right)$ to $\left(h_{2}, h_{2}\right)$. Endowments are indicated within the parentheses in the first column in the tables below. Let $\left\{f_{A(t)}\right\}_{t=1}^{\infty}$ be a constant sequence of period orderings with $\left.f_{A(1)}\right|_{E(1)}:=\left(a_{1}^{-1}, a_{2}^{-1}, a_{3}^{-1}, a_{1}^{0}, a_{2}^{0}, a_{3}^{0}\right)$ and $\left.f_{A(2)}\right|_{E(1)}:=\left(a_{1}^{0}, a_{2}^{0}, a_{3}^{0}\right)$.

\begin{tabular}{|c|c|c|c|}
\hline & $t=1$ & $t=2$ & $\cdots$ \\
\hline$a_{1}^{-1}\left(h_{1}\right)$ & $h_{1}$ & & \\
$a_{2}^{-1}\left(h_{2}\right)$ & $h_{5}$ & & \\
$a_{3}^{-1}\left(h_{3}\right)$ & $h_{3}$ & & \\
\hline$a_{1}^{0}\left(h_{4}\right)$ & $h_{4}$ & $h_{6}$ & \\
$\boldsymbol{a}_{\mathbf{2}}^{\mathbf{0}}\left(h_{5}\right)$ & $\boldsymbol{h}_{\mathbf{2}}$ & $\boldsymbol{h}_{\mathbf{2}}$ & \\
$a_{3}^{0}\left(h_{6}\right)$ & $h_{6}$ & $h_{1}$ & \\
\hline
\end{tabular}

\begin{tabular}{|c|c|c|l|}
\hline & $t=1$ & $t=2$ & $\cdots$ \\
\hline$a_{1}^{-1}\left(h_{1}\right)$ & $h_{1}$ & & \\
$a_{2}^{-1}\left(h_{2}\right)$ & $h_{5}$ & & \\
$a_{3}^{-1}\left(h_{3}\right)$ & $h_{3}$ & & \\
\hline$a_{1}^{0}\left(h_{4}\right)$ & $h_{4}$ & $h_{6}$ & \\
$\boldsymbol{a}_{\mathbf{2}}^{\mathbf{0}}\left(h_{5}\right)$ & $\boldsymbol{h}_{\mathbf{6}}$ & $\boldsymbol{h}_{\mathbf{1}}$ & \\
$a_{3}^{0}\left(h_{6}\right)$ & $h_{2}$ & $h_{2}$ & \\
\hline
\end{tabular}

The left table shows a plan selected by the TTC spot rule when agent $a_{2}^{0}$ reveals her true period preference, while the right table shows a plan selected by the TTC spot rule when $a_{2}^{0}$ lies by reporting the period preference in period 1 and 2 as in the right table above. This shows that $a_{2}^{0}$ manipulates the rule.

For the second part, suppose that two agents arrive in each period. We focus on initial existing tenants in $E(1)=\left\{a_{1}^{-1}, a_{2}^{-1}, a_{1}^{0}, a_{2}^{0}\right\}$. Since there are four initial existing tenants, we explicitly consider four houses $h_{1}$ through $h_{4}$. Suppose all agents $a$ except $a_{2}^{-1}$ have an identical preference such that $P_{a}(t): h_{1}, h_{2}, h_{3}$, and $a_{2}^{-1}$ 's top choice is $h_{4}$. In addition, $\left(h_{2}, h_{2}\right) P_{a_{1}^{0}}\left(h_{3}, h_{1}\right)$ and $\left(h_{3}, h_{1}\right) P_{a_{2}^{0}}\left(h_{2}, h_{2}\right)$. Endowments are indicated in the first column on the table below. Period orderings satisfy $f_{A(t)}=\left(a_{1}^{t-2}, a_{2}^{t-2}, a_{1}^{t-1}, a_{2}^{t-1}, a_{1}^{t}, a_{2}^{t}\right)$ for each $t \geq 1$. The TTC spot rule produces the following plan:

\begin{tabular}{|c|c|c|c|c|}
\hline & $t=1$ & $t=2$ & $t=3$ & $\cdots$ \\
\hline$a_{1}^{-1}\left(h_{1}\right)$ & $h_{1}$ & & & \\
$a_{2}^{-1}\left(h_{2}\right)$ & $h_{4}$ & & & \\
\hline$a_{1}^{0}\left(h_{3}\right)$ & $h_{3}\left(h_{2}\right)$ & $h_{1}\left(h_{2}\right)$ & & \\
$a_{2}^{0}\left(h_{4}\right)$ & $h_{2}\left(h_{3}\right)$ & $h_{2}\left(h_{1}\right)$ & & \\
\hline
\end{tabular}

Consider another plan in which $a_{1}^{0}$ exchange $\left(h_{3}, h_{1}\right)$ for $\left(h_{2}, h_{2}\right)$ with $a_{2}^{0}$. This plan dynamically Pareto dominates the one induced by the TTC spot rule. This shows that the TTC spot rule is not dynamically Pareto efficient.

\section{References}

[1] Abdulkadiroğlu, A., Loertscher, S., 2007. Dynamic house allocations. Working paper.

[2] Abdulkadiroğlu, A., Sönmez, T., 1998. Random serial dictatorship and the core from random endowments in house allocation problems. Econometrica, 66, 689-701.

[3] Abdulkadiroğlu, A., Sönmez, T., 1999. House allocation with existing tenants. J. Econ. Theory, 88, 233-260. 
[4] Abdulkadiroğlu, A., Sönmez, T., 2003. School choice: a mechanism design approach. Amer. Econ. Rev., 93, 729-747.

[5] Athey, S., Segal, I., 2007. An efficient dynamic mechanism. Working paper.

[6] Bloch, F., Cantala, D., 2008. Markovian assignment rules. Woring paper.

[7] Chen, Y., Sönmez, T., 2002. Improving efficiency of on-campus housing: an experimental study. Amer. Econ. Rev., 92, 1669-1686.

[8] Ehlers, L., Klaus, B., 2003. Coalitional strategy-proof and resource-monotonic solutions for multiple assignment problems. Soc. Choice Welf., 21, 265-280.

[9] Ergin, H. I., 2000. Consistency in house allocation problems. J. Math. Econ., 34(1), 77-97.

[10] Frederick, S., Loewenstein, G., O’Donoghue, T., 2002. Time discounting and time preference: a critical review. J. Econ. Lit., Vol.XL, 351-401.

[11] Gershkov, A., Moldovanu, B., 2009. Dynamic revenue maximization with heterogeneous objects: a rule design approach. Amer. Econ. J.: Microeconomics, issue 2, 98-168.

[12] Guillen, P., Kesten, O., 2010. Matching markets with mixed ownership: the case for a real-life assignment mechanism. Working paper.

[13] Hylland, A., Zeckhauser, R., 1979. The efficient allocation of individuals to positions. J. Polit. Economy, 87, 293-314.

[14] Kamijo, Y., Kawasaki, R., 2010. Dynamics, stability, and foresight in the ShapleyScarf housing market. J. Math. Econ., 46, 214-222.

[15] Klaus, B., 2008. The coordinate-wise core for multiple-type housing markets is secondbest incentive compatible, J. Math. Econ., 44, 919-924.

[16] Klaus, B., Miyagawa E., 2001. Strategy-proofness, solidarity, and consistency for multiple assignment problems. Int. J. Game Theory, 30, 421-435.

[17] Konishi, H., Quint, T., Wako, J., 2001. On the Shapley-Scarf economy: the case of multiple types of indivisible goods. J. Math. Econ., 35, 1-15.

[18] Kurino, M., 2009. House allocation with overlapping agents: a dynamic mechanism design approach. Working paper.

[19] Ljungqvist, L., Sargent, T. J., 2004. Recursive Macroeconomic Theory, 2nd edition, MIT press. 
[20] Milgrom, P., Roberts, J., 1992. Economics, Organization and Management, Prentice Hall.

[21] Pápai, S., 2000a. Strategyproof assignment by hierarchical exchange. Econometrica, 68, 1403-1433.

[22] Pápai, S., 2000b. Strategyproof multiple assignment using quotas. Rev. Econ. Des., 5, 91-105.

[23] Pápai, S., 2001. Strategyproof and nonbossy multiple assignments. J. Public Econ. Theory, 3(3), 257-271.

[24] Pycia, M., Ünver, M. U., 2010. Incentive compatible allocation and exchange of discrete resources. Working paper.

[25] Roth, A. E., Sönmez, T., Ünver, M. U., 2004. Kidney exchange. Quart. J. Econ., 119, 457-488.

[26] Roth, A. E., Sönmez, T., Ünver, M.U., 2005. Pairwise kidney exchange. J. Econ. Theory, 125, 151-188.

[27] Samuelson, P. A., 1958. An exact consumption-loan model of interest with or without the social contrivance of money. J. Polit. Economy, 66, 467-482.

[28] Shapley, L., Scarf, H., 1974. On cores and indivisibility. J. Math. Econ., 1, 23-28.

[29] Shell, K., 1971. Notes on the economics of infinity. J. Polit. Economy, 79, 1002-1011.

[30] Sönmez, T., Ünver, M.U., 2005. House allocation with existing tenants: an equivalence. Games Econ. Behav., 52, 153-185.

[31] Sönmez, T., Ünver, M.U., 2010. House allocation with existing tenants: a characterization. Games Econ. Behav., 69(2), 425-445.

[32] Sönmez, T., Ünver, M.U., 2011. Matching, allocation, and exchange of discrete resources. J. Bennhabib, A. Bisin, and M. Jackson (eds.), Handbook of Social Economics, Vol. 1A. The Netherlands: North-Holland, 781-852.

[33] Svensson, L.-G., 1994. Queue allocation of indivisible goods. Soc. Choice Welf., 11, 323-330.

[34] Thomson, W., 2008. Fair allocations rules, K. Arrow, A. Sen, and K. Suzumura (eds.), Handbook of Social Choice and Welfare, Norh-Holland, Amsterdam, New York, forthcoming.

[35] Ünver, M. U., 2010. Dynamic kidney exchange. Rev. Econ. Stud., 77(1), 372-414. 
[36] Wako, J., 2005. Coalition-proof Nash allocation in a barter game with multiple indivisible goods. Math. Soc. Sci., 179-199. 